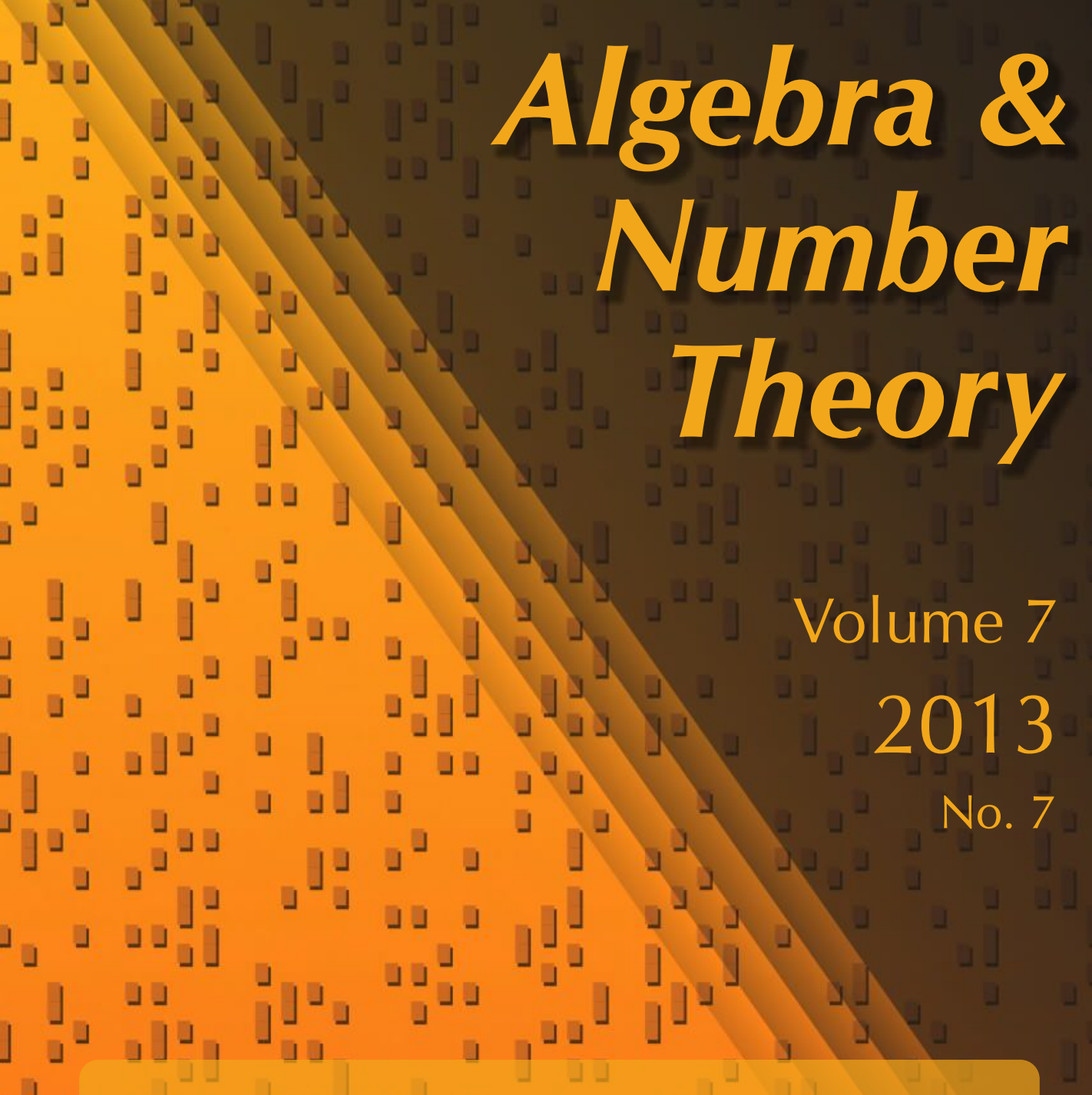

\title{
Weil representation and transfer factor
}

Teruji Thomas

!」

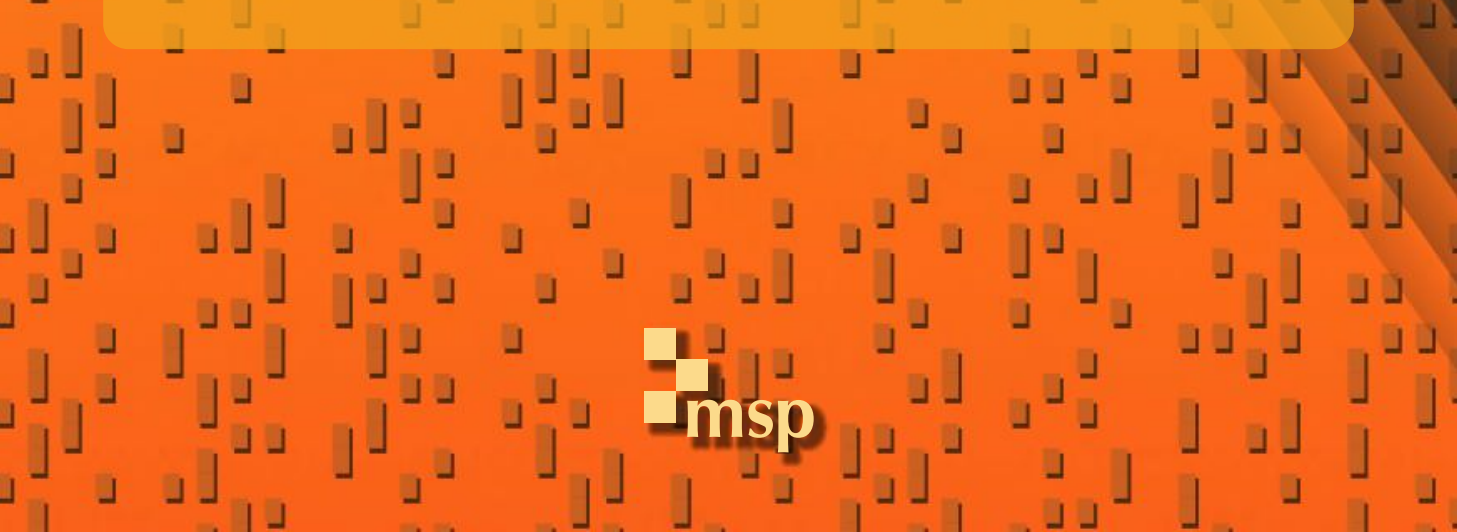




\title{
Weil representation and transfer factor
}

\author{
Teruji Thomas
}

\begin{abstract}
This paper concerns the Weil representation of the semidirect product of the metaplectic and Heisenberg groups. First we present a canonical construction of the metaplectic group as a central extension of the symplectic group by a subquotient of the Witt group. This leads to simple formulas for the character, for the inverse Weyl transform, and for the transfer factor appearing in J. Adams's work on character lifting. Along the way, we give formulas for outer automorphisms of the metaplectic group induced by symplectic similitudes. The approach works uniformly for finite and local fields.
\end{abstract}

\section{Introduction}

1.1. This paper presents some calculations related to the character of the Weil representation. This representation has a fundamental role in the representation theory of the symplectic group and in many related contexts. Before explaining the results, let us recall the classical theory as explained by Lion and Vergne [1980].

Let $V$ be a finite-dimensional vector space, with symplectic form $\omega$. The ground field may be any finite or local field $F$ of characteristic not 2; for example, most classically, $F$ could be the real numbers. Let $\operatorname{Sp}(V)$ be the corresponding symplectic group, that is, the group of automorphisms of $V$ preserving $\omega$. Choose a nontrivial, continuous group homomorphism $\psi: F \rightarrow U(1) \subset \mathbb{C}^{\times}$; for example, in the case of the real numbers, one may take $\psi(x)=e^{i x}$. Choose also a Lagrangian subspace $\ell \subset V$. From the data $(\psi, \ell)$, one constructs a central extension

$$
1 \rightarrow Z_{F} \rightarrow \mathrm{Mp}^{\psi, \ell}(V) \rightarrow \mathrm{Sp}(V) \rightarrow 1 .
$$

$\mathrm{Mp}^{\psi, \ell}(V)$ is known as "the" metaplectic group; as we will see, it is essentially independent of $\psi$ and $\ell$. In the special case when $F$ is $\mathbb{C}$ or a finite field, ${ }^{1}$ the central factor $Z_{F}$ is trivial, so that $\mathrm{Mp}^{\psi, \ell}(V)$ is nothing but the symplectic group $\operatorname{Sp}(V)$; in all other cases, $Z_{F}=Z_{2}=\{ \pm 1\}$, and the extension is nontrivial. For example, when $F=\mathbb{R}, \operatorname{Mp}^{\psi, \ell}(V)$ is the unique connected double cover of $\operatorname{Sp}(V)$.

MSC2010: primary 11F27; secondary 20C15.

Keywords: metaplectic group, Weil representation, Weyl transform, transfer factor, Cayley transform, Maslov index.

${ }^{1}$ See Section 1.5.2 for more simplifications in these cases. 
The construction of $\mathrm{Mp}^{\psi, \ell}(V)$ goes hand-in-hand with the construction of a unitary representation $\rho_{\mathrm{Mp}}^{\psi, \ell}$, known as the Weil representation (also as the oscillator or metaplectic representation). One starts from the Heisenberg group $H(V)$, which is a central extension of $V$ by $F$ as additive groups; thus

$$
H(V)=V \times F \quad(\text { as a set }) .
$$

Associated to the data $(\psi, \ell)$ is an irreducible unitary representation $\rho_{H}^{\psi, \ell}$ of $H(V)$ whose restriction to the center $F \subset H(V)$ is $\psi$ (it is, up to nonunique isomorphism, the unique such representation, but its construction depends also on $\ell$ ). Meanwhile, the natural action of $\operatorname{Sp}(V)$ on $V$ defines a semidirect product $\operatorname{Sp}(V) \ltimes H(V)$. The central extension $\operatorname{Mp}^{\psi, \ell}(V)$ is defined so that $\rho_{H}^{\psi, \ell}$ naturally extends to a representation $\rho^{\psi, \ell}$ of the covering group $\mathrm{Mp}^{\psi, \ell}(V) \ltimes H(V)$. Its restriction to $\mathrm{Mp}^{\psi, \ell}(V)$ is the Weil representation $\rho_{\mathrm{Mp}}^{\psi, \ell}$.

1.2. A number of people have recently studied the character $\operatorname{Tr} \rho^{\psi, \ell}$, defined to be the generalized function on $\mathrm{Mp}^{\psi, \ell}(V) \ltimes H(V)$ whose integral against any smooth, compactly supported measure $h$ on $\operatorname{Mp}^{\psi, \ell}(V) \ltimes H(V)$ is

$$
\int h \cdot \operatorname{Tr} \rho^{\psi, \ell}=\operatorname{Tr}\left(\int h \cdot \rho^{\psi, \ell}\right) .
$$

(The right-hand side is the trace of a trace-class operator - see Remark 5.3.1.) The studies mentioned make some restrictions, focusing on $\mathrm{Mp}^{\psi, \ell}(V)$ (e.g., [Thomas 2008]), or on some open subset (e.g., [Maktouf 1999; Gurevich and Hadani 2007]), and/or making a particular choice of field (e.g., [de Gosson and Luef 2009] for the reals, [Gurevich and Hadani 2007; Prasad 2009] for finite fields). This article completes the project in the following ways.

(A) The different metaplectic groups $\mathrm{Mp}^{\psi, \ell}$ corresponding to varying data $(\psi, \ell)$ are canonically isomorphic. The first task is to construct an extension

$$
1 \rightarrow Z_{F} \rightarrow \mathrm{Mp}(V) \rightarrow \mathrm{Sp}(V) \rightarrow 1
$$

isomorphic to (1), but defined without any reference to $\psi$ and $\ell$. Using this canonical construction, we give explicit formulas for the isomorphisms between the various groups $\mathrm{Mp}^{\psi, \ell}(V)$. As a by-product, we find explicit formulas for the conjugation action of $\operatorname{GSp}(V)$ on $\mathrm{Mp}(V)$ and $\mathrm{Mp}^{\psi, \ell}(V)$.

(B) Because of (A), every Weil representation $\rho^{\psi, \ell}$ can be considered as a representation of the single group $\operatorname{Mp}(V) \ltimes H(V)$. We give a formula for the character $\operatorname{Tr} \rho^{\psi, \ell}$ as a generalized function on $\operatorname{Mp}(V) \ltimes H(V)$. The isomorphisms described in (A) allow easy translation of this character formula to other versions of the metaplectic group. 
(C) The answer to (B) also yields explicit formulas for the "invariant presentation", or inverse Weyl transform, of $\rho_{\mathrm{Mp}}^{\psi, \ell}$; this is (roughly speaking) a homomorphism from $\mathrm{Mp}(V)$ into the $\psi$-coinvariant group algebra of $H(V)$.

(D) Writing $\rho_{\mathrm{Mp}}^{\psi, \ell}=\rho_{+}^{\psi, \ell} \oplus \rho_{-}^{\psi, \ell}$ as the direct sum of two irreducibles, we calculate the character of the virtual representation $\rho_{+}^{\psi, \ell}-\rho_{-}^{\psi, \ell}$ (which then determines the characters of $\rho_{+}^{\psi, \ell}$ and $\rho_{-}^{\psi, \ell}$ separately). This is a generalized function on $\operatorname{Mp}(V)$. Over a finite field, the method leads naturally to a "geometric" version of this virtual character, in the sense of Grothendieck's sheaf-function dictionary.

The virtual character in (D) plays a key role in Jeff Adams's theory [1998] of character lifting between metaplectic and orthogonal groups, which provides one of my main motivations for studying this subject.

Remark 1.2.1. The method for (B) is closely related to Roger Howe's wonderful unpublished notes [1973], and some similar ideas have been exploited by Gurevich and Hadani [2007] over finite fields, and de Gosson and Luef [2009] over the reals. In particular, the work of de Gosson ([op. cit.] and references therein) gives a very nice, and closely related, character formula in terms of the Conley-Zehnder index of paths in the real symplectic group.

1.3. Results. (A) The construction of the canonical metaplectic extension (3) proceeds in two steps, which make sense for any field $F$ of characteristic not 2 . The details are given in Section 2; here we outline the basic features, to fix our notation. First we define a central extension

$$
0 \rightarrow W(F) / I^{3} \rightarrow M(V) \rightarrow \mathrm{Sp}(V) \rightarrow 1
$$

where $W(F)$ is the Witt ring of quadratic spaces over $F$, and $I \subset W(F)$ is the ideal of even-dimensional quadratic spaces (see Section A.1 in the Appendix). This construction is by means of a cocycle, so that

$$
M(V)=\operatorname{Sp}(V) \times W(F) / I^{3} \quad \text { as a set. }
$$

Second, we define $\operatorname{Mp}(V)$ to be a certain subgroup of $M(V)$. In short, $\operatorname{Mp}(V)$ is the unique subgroup extending $\operatorname{Sp}(V)$ by $I^{2} / I^{3}$ :

$$
0 \rightarrow I^{2} / I^{3} \rightarrow \mathrm{Mp}(V) \rightarrow \mathrm{Sp}(V) \rightarrow 1 .
$$

It turns out (see Theorem A.2) that, for a finite or local field, we can identify $I^{2} / I^{3}$ with the group $Z_{F}$, thus obtaining (3) as a special case. Concretely, for each $g \in \mathrm{Sp}(V)$, define a bilinear form $\sigma_{g}$ on $(g-1) V$ by the formula

$$
\sigma_{g}((g-1) x,(g-1) y)=\omega(x,(g-1) y) \quad \text { for all } x, y \in V .
$$


Then $\sigma_{g}$ is nondegenerate as a bilinear form, but, in general, asymmetric. It nonetheless has a rank $\operatorname{dim} \sigma_{g}=\operatorname{dim}(g-1) V$ and discriminant $\operatorname{det} \sigma_{g} \in F^{\times} /\left(F^{\times}\right)^{2}$. This is enough to determine a class $\left[\sigma_{g}\right]$ in $W(F) / I^{2}$ — the class of quadratic spaces with the same rank modulo 2 and the same signed discriminant as $\sigma_{g}$ (see Section A.1). The definition of $\operatorname{Mp}(V)$ is as follows:

$$
\operatorname{Mp}(V)=\left\{(g, q) \in M(V) \mid q=\left[\sigma_{g}\right] \bmod I^{2} / I^{3}\right\} .
$$

In Proposition 2.4 we show that this definition makes $\mathrm{Mp}(V)$ into a subgroup of $M(V)$, and therefore obviously an extension of $\operatorname{Sp}(V)$ by $I^{2} / I^{3}$.

In Section 2.6 we also recall the construction of $\mathrm{Mp}^{\psi, \ell}(V)$ from [Lion and Vergne 1980] — this construction requires $F$ to be finite or local. In Section 3 we describe canonical isomorphisms $\mathrm{Mp}(V) \rightarrow \mathrm{Mp}^{\psi, \ell}(V)$. They are "canonical" in the sense of being unique as isomorphisms of central extensions; see Section 3.1.

Remark 1.3.1. The idea of constructing an extension by $I^{2} / I^{3}$ comes from [Parimala et al. 2000] (using, however, a choice of Lagrangian $\ell \subset V$; see Section 2.7.1 for a synopsis). It also follows from the work of Suslin [1987] that these extensions can be characterized by a universal property; see Remark 2.5.1.

Remark 1.3.2. The Weil representation (which, again, is defined only when $F$ is a finite or local field) can be extended very naturally to a representation of $M(V)$ rather than $\operatorname{Mp}(V)$, and practically all the results stated herein for $\operatorname{Mp}(V)$ hold also for $M(V)$. However, we will continue to refer primarily to $\mathrm{Mp}(V)$, to connect better with the literature.

(B) For the rest of this introduction, we take $F$ to be a finite or local field, so that $\rho^{\psi, \ell}$ is defined (we recall the definition in Section 4). We consider it as a representation of $\operatorname{Mp}(V) \ltimes H(V)$. To describe its character, we need some further notation.

Notation. Let $\gamma_{\psi}: W(F) / I^{3} \rightarrow \mathbb{C}^{\times}$be the Weil index (see Section A.3, especially A.4.1(d)). For any $g \in \operatorname{Sp}(V)$, let $Q_{g}$ be the associated Cayley form: it is a symmetric, usually degenerate, bilinear form on $(g-1) V$ defined by

$$
Q_{g}((g-1) x,(g-1) y):=\frac{1}{2} \omega((g+1) x,(g-1) y) \text { for all } x, y \in V .
$$

Some further comments about the Cayley form are given in Section A.6.

Finally, let $\mu_{\sigma_{g}}$ be the Haar measure on $(g-1) V$ self-dual with respect to $\psi \circ \sigma_{g}$, and $\mu_{V}$ the Haar measure on $V$ self-dual with respect to $\psi \circ \omega$ (see Section A.3.1 for conventions on measures). Define a generalized function $D_{g}^{\psi}$ on $V$ by the equation

$$
\int_{V} f D_{g}^{\psi} \mu_{V}=\int_{(g-1) V} f \mu_{\sigma_{g}}
$$

for all compactly supported, smooth functions $f$ on $V$. 
If $F$ is a finite field, then this definition amounts to the following: $D_{g}^{\psi}$ is the function on $V$ supported on $(g-1) V$ and equal there to the constant $\sqrt{\# \operatorname{ker}(g-1)}$. When $F$ is infinite, we just have $D_{g}^{\psi}(v)=\|\operatorname{det}(g-1)\|^{-1 / 2}$ if $\operatorname{det}(g-1) \neq 0$ (and, as standard, we choose the norm $\|\cdot\|$ on $F^{\times}$such that $d(a x)=\|a\| d x$ for any translation-invariant measure $d x$ on $F$ ).

Theorem B (character formula). For fixed $(g, q) \in \mathrm{Mp}(V)$, the character

$$
T_{(g, q)}^{\psi}(v, t):=\operatorname{Tr} \rho^{\psi, \ell}(g, q ; v, t)
$$

is a well-defined generalized function of $(v, t) \in H(V)$, supported on $(g-1) V \times F$, and given by

$$
T_{(g, q)}^{\psi}(v, t)=\psi\left(\frac{1}{2} Q_{g}(v, v)\right) \cdot D_{g}^{\psi}(v) \cdot \gamma_{\psi}(q) \cdot \psi(t) .
$$

The main part of the proof, using the Weyl transform, is given in Section 5. Note that the right-hand side is manifestly independent of $\ell$, reflecting the independence of $\rho^{\psi, \ell}$ up to nonunique isomorphism.

Theorem B can be read as a formula for a locally integrable function ${ }^{2}$ on

$$
\operatorname{Mp}(V) \ltimes H(V)
$$

representing $\operatorname{Tr} \rho^{\psi, \ell}$, but it says something more precise. The point is that, when $F$ is infinite, $\operatorname{Tr} \rho^{\psi, \ell}$ is smooth almost everywhere, but "blows up" on the locus where $\operatorname{det}(g-1)=0$. Theorem B gives a natural extension of $\operatorname{Tr} \rho^{\psi, \ell}$ to that singular locus - "natural" in the sense that it satisfies Theorem $\mathrm{C}$ below.

If we are only interested in the representation $\rho_{\mathrm{Mp}}^{\psi, \ell}$ of $\mathrm{Mp}(V)$ then Theorem B takes on the following simple form. Let

$$
D^{0}(g):=\sqrt{\# V^{g}} \text { or } \quad D^{0}(g):=\|\operatorname{det}(g-1)\|^{-1 / 2}
$$

depending on whether $F$ is finite or infinite. Here $V^{g}:=\operatorname{ker}(g-1)$.

Corollary 1.4 (restriction to $\mathrm{Mp}(V)$ ). As generalized functions of $(g, q) \in \mathrm{Mp}(V)$,

$$
\operatorname{Tr} \rho_{\mathrm{Mp}}^{\psi, \ell}(g, q)=D^{0}(g) \cdot \gamma_{\psi}(q) .
$$

The extreme simplicity of this formula suggests that the cocycle we have used to define $\operatorname{Mp}(V)$ is the natural one in this context. In particular, it is much better than the formula we developed in [Thomas 2008]. (In Remark 2.8.2 we explain how the thing called $\mathrm{Mp}(V)$ in that work is related to the present one.)

${ }^{2}$ That is, for $F$ infinite, $D_{g}^{\psi}(v)=\|\operatorname{det}(g-1)\|^{-1 / 2}$ for almost all $(g, v) \in \operatorname{Sp}(V) \times V$, and $(g, q, v, t) \mapsto \psi\left(\frac{1}{2} Q_{g}(v, v)\right) \cdot\|\operatorname{det}(g-1)\|^{-1 / 2} \cdot \gamma_{\psi}(q) \cdot \psi(t)$ is locally integrable on $\operatorname{Mp}(V) \ltimes H(V)$ : the modulus is just $\|\operatorname{det}(g-1)\|^{-1 / 2}$, so that the singularities of order $k / 2$ lie in subspaces of codimension at least $k$. 
(C) The formula of Theorem B also makes explicit the "invariant presentation" of the Weil representation emphasized, for example, in [Gurevich and Hadani 2007]. Let us recall that description. Let $\mathscr{A}_{\psi}$ be the $L^{2}$-completion of the $\psi$-coinvariant group-algebra of $H(V)$. In more detail, we consider functions on $H(V)$ that transform by $\psi$ under the action of the center $F \subset H(V)$; these can be identified (by restriction) with functions on $V$. With that in mind, we define $\mathscr{A}_{\psi}$ to consist of all complex $L^{2}$ functions on $V$, equipped with the "convolution" multiplication induced by the multiplication on $H(V)$ :

$$
\left(f_{1} \star f_{2}\right)(x):=\int_{v \in V} f_{1}(v) \psi\left(\frac{1}{2} \omega(v, x)\right) f_{2}(x-v) \mu_{V} .
$$

(Here $\mu_{V}$ again denotes the Haar measure on $\mu_{V}$ that is self-dual with respect to $\psi \circ \omega$.$) It is well known, and we prove in Proposition 5.2, that there is an$ isomorphism $W^{\psi, \ell}$ from $\mathscr{A}_{\psi}$ to the algebra of Hilbert-Schmidt operators on the representation space of $\rho^{\psi, \ell}$. This $W^{\psi, \ell}$ is called the Weyl transform.

Theorem C. For any $f \in \mathscr{A}_{\psi}$, the convolution $T_{(g, q)}^{\psi} \star f$ is well-defined and lies in $A_{\psi}$, and

$$
W^{\psi, \ell}\left(T_{(g, q)}^{\psi} \star f\right)=\rho^{\psi, \ell}(g, q) \circ W^{\psi, \ell}(f) .
$$

Theorem $\mathrm{C}$ may be restated more transparently when $F$ is a finite field: it says that the map $(g, q) \mapsto T_{(g, q)}^{\psi}$ is a multiplicative homomorphism $\operatorname{Mp}(V) \rightarrow \mathscr{A}_{\psi}$, and $W^{\psi, \ell}\left(T_{(g, q)}^{\psi}\right)=\rho^{\psi, \ell}(g, q)$.

Versions of Theorem $\mathrm{C}$ are well known (see for example [Gurevich and Hadani 2007, §1.2; Howe 1973, Theorem 2.9]), so the new aspect is the explicit formula provided by Theorem B; nonetheless, we will find it convenient and easy to prove Theorem C in Section 6.

(D) The representation space of $\rho^{\psi, \ell}$ can be understood as the space of $L^{2}$ functions on $V / \ell$. One has a decomposition

$$
\rho_{\mathrm{Mp}}^{\psi, \ell}=\rho_{+}^{\psi, \ell} \oplus \rho_{-}^{\psi, \ell}
$$

into irreducibles, where $\rho_{+}^{\psi, \ell}$ acts on the subspace of even functions, and $\rho_{-}^{\psi, \ell}$ on the subspace of odd ones. In Section 7 we give two proofs of the following result.

Theorem D. As generalized functions of $(g, q) \in \mathrm{Mp}(V)$,

$$
\operatorname{Tr}\left(\rho_{+}^{\psi, \ell}-\rho_{-}^{\psi, \ell}\right)(g, q)=\gamma_{\psi}\left(Q_{g}\right) \cdot \operatorname{Tr} \rho_{\mathrm{Mp}}^{\psi, \ell}(-g, q) .
$$

Again, the right-hand side in Theorem $\mathrm{D}$ is manifestly independent of $\ell$.

Remark 1.4.1. One knows on general grounds that the characters $\operatorname{Tr} \rho_{ \pm}^{\psi, \ell}$ are welldefined (see [Harish-Chandra 1954] for the real case and [Sliman 1984, Theorem 1.2.3] for admissibility in the nonarchimedean case). 
Geometrization. Suppose that $F=\mathbb{F}_{q}$ is a finite field. ${ }^{3}$ In this situation, the central extension (7) is split, so that we may consider $\rho^{\psi, \ell}$ as a representation of $\operatorname{Sp}(V) \ltimes H(V)$. We can also consider $\operatorname{Sp}(V) \ltimes H(V)$ as the $\mathbb{F}_{q}$-points of a group scheme $\mathbb{G}=\mathbb{S p}(V) \ltimes \mathbb{H}(V)$. Gurevich and Hadani [2007] have constructed an irreducible perverse sheaf $\mathscr{K}$ on $\mathbb{G}$ corresponding (under Grothendieck's sheaffunction dictionary) to the character $\operatorname{Tr} \rho^{\psi, \ell}$. The proof of Theorem D (specifically (33)) shows that there is, as well, an irreducible perverse sheaf $\mathscr{K}^{\prime}$ on $\mathbb{G}$ whose pullback to $\operatorname{Sp}(V)$ corresponds to the virtual character $\operatorname{Tr}\left(\rho_{+}^{\psi, \ell}-\rho_{-}^{\psi, \ell}\right)$; namely, $\mathscr{K}^{\prime}$ is just the Fourier-Deligne transform of $\mathscr{K}$ along $V$ with respect to the pairing $\psi \circ \frac{1}{2} \omega$.

Remark 1.4.2. The fact (33) that $\operatorname{Tr}\left(\rho_{+}^{\psi, \ell}-\rho_{-}^{\psi, \ell}\right)$ is related to $\operatorname{Tr} \rho^{\psi, \ell}$ by a Fourier transform explains the relationship between Theorem B and Theorem D: recall (Theorem A.4) that the $\gamma_{\psi}\left(Q_{g}\right)$ appearing in Theorem $\mathrm{D}$ is itself related by Fourier transform to the $\psi \circ \frac{1}{2} Q_{g}$ appearing in Theorem B.

\subsection{Remarks.}

1.5.1. Dependence on $\psi$. Let us briefly clarify the dependence of our results on the character $\psi$. For any chosen $\psi$, any other nontrivial additive character is uniquely of the form $\psi_{a}(x)=\psi(a x)$, with $a \in F^{\times}$. The isomorphism class of $\rho_{\mathrm{Mp}}^{\psi_{a}, \ell}$ depends only on the class of $a$ modulo $\left(F^{\times}\right)^{2}$. For $(g, q) \in \mathrm{Mp}(V)$, we have

$$
\gamma_{\psi_{a}}(q)=\gamma_{\psi}(q) \cdot\left(\gamma_{\psi}(a) / \gamma_{\psi}(1)\right)^{\operatorname{dim}(g-1) V}\left(a, \operatorname{det} \sigma_{g}\right)_{H}
$$

where $(\cdot, \cdot)_{H}$ is the Hilbert symbol (see Lemma 3.13 and Section A.1.2). Moreover, $D_{g}^{\psi_{a}}=D_{g}^{\psi} \cdot\|a\|^{-\left(\operatorname{dim} V^{g}\right) / 2}($ see Section A.3.1).

1.5.2. Special fields. The framework presented here gives a uniform treatment for any choice of field $F$. However, some simplifications are possible, case by case.

When $F=\mathbb{C}$, the central factor $Z_{F}$ is trivial, and both $\gamma_{\psi}$ and the Hilbert symbol always equal 1 . When $F$ is finite, $Z_{F}$ is again trivial. This means that for each $g$, there is a unique $q \in W(F) / I^{3}$ with $(g, q) \in \mathrm{Mp}(V)$. One has

$$
\gamma_{\psi}(q)=\gamma_{\psi}(1)^{\operatorname{dim}(g-1) V-1} \gamma_{\psi}\left(\operatorname{det} \sigma_{g}\right) .
$$

Moreover, the Hilbert symbol always equals $1, \gamma_{\psi}$ takes values in the fourth roots of unity $Z_{4}$ (or even $Z_{2}$ if -1 is a square), and the common expression $\gamma_{\psi}(a) / \gamma_{\psi}(1)$ equals 1 if $a$ is a square, and -1 if not.

\section{Metaplectic cocycles}

In this section we construct the canonical metaplectic extension (4), which exists for any field of characteristic not 2 . We also recall the traditional construction (1)

${ }^{3}$ Lafforgue and Lysenko [2009] have also considered a geometric version of the even part of the Weil representation over a local field $\mathbb{F}_{q}((t))$. 
in Section 2.6, which makes sense only for a finite or local field, and depends on the choice of a Lagrangian $\ell$ and a character $\psi$. In Section 2.7 we examine these choices more closely. This will allow us to give explicit isomorphisms between all these various incarnations of the metaplectic group in Section 3.

The key tools are the Maslov index $\tau$ and the Weil index $\gamma_{\psi}$. The relevant facts and notation concerning these objects are recalled in Appendix A.

2.1. Generalities. Suppose that $G$ is a group and $A$ an abelian group, written additively; by a 2-cocycle $c: G \times G \rightarrow A$ we mean a function such that

$$
c\left(g, g^{\prime}\right)-c\left(g, g^{\prime} g^{\prime \prime}\right)+c\left(g g^{\prime}, g^{\prime \prime}\right)-c\left(g^{\prime}, g^{\prime \prime}\right)=0 \quad \text { and } \quad c(1,1)=0 .
$$

Given such a 2-cocycle, define $\tilde{G}=G \times A$ as a set, with a multiplication operation

$$
(g, a)\left(g^{\prime}, a^{\prime}\right):=\left(g g^{\prime}, a+a^{\prime}+c\left(g, g^{\prime}\right)\right) .
$$

Then it follows from (6) that $\tilde{G}$ is a group, with $A$ as a central subgroup, and $G=\tilde{G} / A$. In other words, we have constructed a central extension

$$
0 \rightarrow A \rightarrow \tilde{G} \rightarrow G \rightarrow 1 \text {. }
$$

Now let us apply this construction to various 2-cocycles, with $G=\operatorname{Sp}(V)$.

2.2. The canonical cocycle. Here we allow $F$ to be any field (but always of characteristic not 2). Let $\bar{V}$ be the symplectic vector space $(V,-\omega)$. Then for each $g \in \operatorname{Sp}(V)$, the graph $\Gamma_{g}=\{(x, g x) \in \bar{V} \oplus V\}$ is a Lagrangian subspace of $\bar{V} \oplus V$. Define

$$
c(g, h)=\tau\left(\Gamma_{1}, \Gamma_{g}, \Gamma_{g h}\right)
$$

for $g, h \in \operatorname{Sp}(V)$.

Lemma 2.3. The function $c: G \times G \rightarrow W(F)$ is a 2-cocycle.

Proof. The left-hand side of (6) is

$$
\tau\left(\Gamma_{1}, \Gamma_{g}, \Gamma_{g g^{\prime}}\right)-\tau\left(\Gamma_{1}, \Gamma_{g}, \Gamma_{g g^{\prime} g^{\prime \prime}}\right)+\tau\left(\Gamma_{1}, \Gamma_{g g^{\prime}}, \Gamma_{g g^{\prime} g^{\prime \prime}}\right)-\tau\left(\Gamma_{1}, \Gamma_{g^{\prime}}, \Gamma_{g^{\prime} g^{\prime \prime}}\right) .
$$

The last term is $-\tau\left(\Gamma_{g}, \Gamma_{g g^{\prime}}, \Gamma_{g g^{\prime} g^{\prime \prime}}\right)$, applying A.5(d) to $1 \oplus g \in \mathrm{GL}(\bar{V} \oplus V)$. Thus the sum is a sum over the faces of the following tetrahedron, with each face contributing the Maslov index of its vertices, in the manner explained in Section A.5.2.

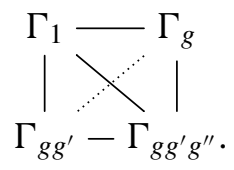

The sum therefore vanishes. 
From now on we reduce the values of $c$ modulo $I^{3}$, where (as explained in Section A.1), $I \subset W(F)$ is the ideal of even-dimensional quadratic spaces. ${ }^{4}$ Thus we obtain the following definition.

Definition 2.3.1. Let $M(V)$ be the central extension

$$
0 \rightarrow W(F) / I^{3} \rightarrow M(V) \rightarrow \mathrm{Sp}(V) \rightarrow 1
$$

defined by the cocycle $c$.

Remark 2.3.2. When $F$ is a local field, $M(V)$ has a natural topology, as described in Remark 2.8.3 below.

2.3.3. Reduction to $I^{2} / I^{3}$. We now construct $\operatorname{Mp}(V)$ as a subgroup of $M(V)$, fitting into a central extension

$$
0 \rightarrow I^{2} / I^{3} \rightarrow \mathrm{Mp}(V) \rightarrow \mathrm{Sp}(V) \rightarrow 1 .
$$

When $F$ is a finite or local field, $I^{2} / I^{3}=Z_{F}$ (see Theorem A.2), yielding the central extension (3).

Definition 2.3.4. Let $\sigma_{g}$ be the nondegenerate bilinear form on $(g-1) V$ defined $^{5}$ by

$$
\sigma_{g}((g-1) x,(g-1) y)=\omega(x,(g-1) y) \quad \text { for all } x, y \in V .
$$

Let $\left[\sigma_{g}\right]$ be the class in $W(F) / I^{2}$ generated by quadratic spaces with the same rank mod 2 and the same signed discriminant as $\sigma_{g}$; see Remark A.1.1. Let $\operatorname{Mp}(V) \subset M(V)$ be the subset of pairs $(g, q)$ such that $q=\left[\sigma_{g}\right] \bmod I^{2} / I^{3}$.

We will have constructed a central extension (8) if we can prove this:

Proposition 2.4. $\mathrm{Mp}(V)$ is a subgroup of $M(V)$.

Proof. We use the calculation of the rank and discriminant of the Maslov index described in Section A.5.1. Write $\alpha_{g}=(1, g): \Gamma_{1} \rightarrow \Gamma_{g}$. Choose a nonzero $o_{1} \in \operatorname{det}\left(\Gamma_{1}\right)$, and let $o_{g}=\alpha_{g}\left(o_{1}\right) \in \operatorname{det}\left(\Gamma_{g}\right)$. Let us calculate $Q\left(\Gamma_{g}, o_{g} ; \Gamma_{1}, o_{1}\right)$, as defined in Section A.5.1. Using $\alpha=\alpha_{g}^{-1}$, this is the class in $W(F) / I^{2}$ of the bilinear form

$$
q(x, g x ; y, g y)=\omega(x, g y)-\omega(x, y)=\omega(x,(g-1) y)
$$

\footnotetext{
${ }^{4}$ The reduction modulo $I^{3}$ is not crucial. We could deal with extensions of $\operatorname{Sp}(V)$ by $W(F)$ and $I^{2}$ rather than $W(F) / I^{3}$ and (as below) $I^{2} / I^{3}$. However, it is convenient that for finite and local fields, we can identify $I^{2} / I^{3}$ with the group $Z_{F}$ (see Theorem A.2; in fact, $I^{3}=0$ for all finite or local fields other than $\mathbb{R}$ ). The reduction modulo $I^{3}$ is also necessary for Proposition 3.16 .

${ }^{5}$ To see that $\sigma_{g}$ is well defined, suppose that $(g-1) x=0$. The claim is that $\omega(x,(g-1) y)=0$. By direct calculation, $\omega(x,(g-1) y)=-\omega((g-1) x, g y)=-\omega(0, g y)=0$. To see that $\sigma_{g}$ is nondegenerate, observe that if, for some $(g-1) y$ and all $(g-1) x, \sigma_{g}((g-1) x,(g-1) y)=0$, then $\omega(x,(g-1) y)=0$ for all $x$, whence $(g-1) y=0$ by the nondegeneracy of $\omega$.
} 
pairing $(x, g x)$ and $(y, g y) \in \Gamma_{g} / \Gamma_{g} \cap \Gamma_{1}$. But $(x, g x) \mapsto(g-1) x$ is an isometry between $\left(\Gamma_{g} / \Gamma_{g} \cap \Gamma_{1}, q\right)$ and $\left((g-1) V, \sigma_{g}\right)$. Therefore

$$
Q\left(\Gamma_{g}, o_{g} ; \Gamma_{1}, o_{1}\right)=\left[\sigma_{g}\right] \in W(F) / I^{2} .
$$

Now, according to (34) and the preceding discussion,

$$
\begin{aligned}
\tau\left(\Gamma_{1}, \Gamma_{g}, \Gamma_{g g^{\prime}}\right) & =Q\left(\Gamma_{g g^{\prime}}, o_{g g^{\prime}} ; \Gamma_{1}, o_{1}\right)+Q\left(\Gamma_{1}, o_{1} ; \Gamma_{g}, o_{g}\right)+Q\left(\Gamma_{g}, o_{g} ; \Gamma_{g g^{\prime}}, o_{g g^{\prime}}\right) \\
& =Q\left(\Gamma_{g g^{\prime}}, o_{g g^{\prime}} ; \Gamma_{1}, o_{1}\right)-Q\left(\Gamma_{g}, o_{g} ; \Gamma_{1}, o_{1}\right)-Q\left(\Gamma_{g^{\prime}}, o_{g^{\prime}} ; \Gamma_{1}, o_{1}\right)
\end{aligned}
$$

(all modulo $I^{2}$ ) and therefore, by our calculation,

$$
\tau\left(\Gamma_{1}, \Gamma_{g}, \Gamma_{g g^{\prime}}\right)=\left[\sigma_{g g^{\prime}}\right]-\left[\sigma_{g^{\prime}}\right]-\left[\sigma_{g}\right] \bmod I^{2} .
$$

This is exactly the condition for $\operatorname{Mp}(V)$ to be closed under multiplication.

2.4.1. Uniqueness. Before proceeding, note that in fact $\operatorname{Mp}(V)$ is the unique subgroup of $M(V)$ such that the projection to $\operatorname{Sp}(V)$ makes it a central extension of $\operatorname{Sp}(V)$ by $I^{2} / I^{3}$. Indeed, the following general statement applies.

Lemma 2.5. Suppose that $\tilde{G}$ is a central extension of $\operatorname{Sp}(V)$ by an abelian group $A$. For any subgroup $B \subset A$ such that $A / B$ has no 3-torsion, there is at most one subgroup $\tilde{G}^{\prime} \subset \tilde{G}$ such that the given projection $\tilde{G}^{\prime} \rightarrow \operatorname{Sp}(V)$ is surjective with kernel B.

In our case, $A=W(F) / I^{3}$ and $B=I^{2} / I^{3}$; the lemma applies because $A / B=$ $W(F) / I^{2}$ has only 2-primary torsion (being isomorphic to the group $W_{0}(F)$ described in Section A.1). In fact, $W(F)$ itself, and therefore any subquotient, has only 2-primary torsion; see [Lam 2005, Chapter 8, Theorem 3.2].

Proof of Lemma 2.5. Suppose that $\tilde{G}^{\prime}$ and $\tilde{G}^{\prime \prime}$ are two such subgroups. Then for each $g \in \operatorname{Sp}(V)$ there exists $f(g) \in A$ such that $(g, a) \in \tilde{G}^{\prime} \Longleftrightarrow(g, a+f(g)) \in \tilde{G}^{\prime \prime}$. Moreover, $f(g)$ is unique modulo $B$, and $f$ is a homomorphism $\operatorname{Sp}(V) \rightarrow A / B$. Thus it is enough to prove that there is no nontrivial homomorphism $\operatorname{Sp}(V) \rightarrow A / B$. In fact, $\operatorname{Sp}(V)$ is perfect unless $V \cong \mathbb{F}_{3}^{2}$; see [Grove 2001, Propositions 3.7-3.8]. In that exceptional case, the abelianization of $\operatorname{Sp}(V)$ is cyclic of order 3 (one can compute that $\operatorname{Sp}(V) \cong \mathrm{SL}_{2}\left(\mathbb{F}_{3}\right)$ has 24 elements, and that the commutator subgroup is the unique subgroup of order 8). Since, by assumption, $A / B$ has no 3-torsion, any homomorphism $\operatorname{Sp}(V) \rightarrow A$ is trivial.

Remark 2.5.1. The metaplectic extension $\operatorname{Mp}(V)$ of $\operatorname{Sp}(V)$ by $I^{2} / I^{3}$ also has a universal property, which can be deduced from the work of Suslin [1987]. Namely, the metaplectic extension of $\operatorname{Sp}_{2 n}(F)$ is the universal central extension that extends to $\mathrm{SL}_{2 n}(F)$ and splits over $\mathrm{SL}_{n}(F)$. 
2.6. The traditional cocycle. Now we assume that $F$ is finite or local, which allows us to use the Weil index $\gamma_{\psi}$ (see Section A.3).

That is, for chosen Lagrangian subspace $\ell \subset V$ and nontrivial additive character $\psi: F \rightarrow \mathbb{C}^{\times}$, define

$$
c_{\psi, \ell}\left(g, g^{\prime}\right)=\gamma_{\psi}\left(\tau\left(\ell, g \ell, g g^{\prime} \ell\right)\right) .
$$

Then $c_{\psi, \ell}$ is a 2-cocycle with values in the group $Z_{8} \subset \mathbb{C}^{\times}$of eighth roots of unity (as can be proved in parallel to Lemma 2.3).

Definition 2.6.1. Define a central extension

$$
1 \rightarrow Z_{8} \rightarrow M^{\psi, \ell}(V) \rightarrow \mathrm{Sp}(V) \rightarrow 1
$$

using the cocycle $c_{\psi, \ell}$.

2.6.2. Reduction to $Z_{F}$. We now construct $\mathrm{Mp}^{\psi, \ell}(V)$ as a subgroup of $M^{\psi, \ell}(V)$, fitting into a central extension

$$
1 \rightarrow Z_{F} \rightarrow \operatorname{Mp}^{\psi, \ell}(V) \rightarrow \mathrm{Sp}(V) \rightarrow 1 .
$$

We use the notation of Section A.5.1. Choose an orientation $o \in \operatorname{det}(\ell)$, and, for each $g \in \mathrm{Sp}(V)$, let $g o$ be the corresponding orientation of $g \ell$. The class $Q(g \ell, g o ; \ell, o) \in W(F) / I^{2}$ is independent of the choice of $o$.

Definition 2.6.3. Let $\mathrm{Mp}^{\psi, \ell}(V) \subset M^{\psi, \ell}(V)$ be the subset of pairs $(g, \xi)$ with

$$
\xi=\gamma_{\psi}(Q(g \ell, g o ; \ell, o)) \bmod Z_{F} .
$$

(Recall that $Q(g \ell, g o ; \ell, o)$ is defined modulo $I^{2}$, and that $\gamma_{\psi}\left(I^{2}\right)=Z_{F}$; see Property A.4.1(d).)

It follows easily from (34) that $\mathrm{Mp}^{\psi, \ell}(V)$ is a subgroup of $M^{\psi, \ell}(V)$; indeed, by Lemma 2.5 , it is the unique subgroup yielding a central extension of $\operatorname{Sp}(V)$ by $Z_{F}$.

Remark 2.6.4. The definition of $\mathrm{Mp}^{\psi, \ell}(V)$ can be unwound a bit to give a standard formula, as follows. For each $g \in \operatorname{Sp}(V)$, choose a basis $\left(q_{1}, \ldots, q_{n}\right)$ of $\ell$ and a basis $\left(p_{1}, \ldots, p_{m}, q_{m+1}, \ldots, q_{n}\right)$ of $g \ell$, such that $\left(q_{m+1}, \ldots, q_{n}\right)$ is a basis for $\ell \cap g \ell$ and $\omega\left(p_{i}, q_{j}\right)=\delta_{i j}$. Let $\theta^{\ell}(g) \in F^{\times}$be the scalar such that

$$
g q_{q} \wedge \cdots \wedge g q_{n}=\theta^{\ell}(g)\left(p_{1} \wedge \cdots \wedge p_{m} \wedge q_{m+1} \wedge \cdots \wedge q_{n}\right)
$$

in $\operatorname{det}(g \ell)$. The class of $\theta^{\ell}(g)$ in $F^{\times} /\left(F^{\times}\right)^{2}$ is independent of the bases. Then $\operatorname{Mp}^{\psi, \ell}(V) \subset M^{\psi, \ell}(V)$ is the subset of pairs $(g, \xi)$ with

$$
\xi=\gamma_{\psi}(1)^{\operatorname{dim}(\ell / \ell \cap g \ell)-1} \gamma_{\psi}\left(\theta^{\ell}(g)\right) \bmod Z_{F} .
$$

Indeed, this follows from Section A.4.1(c): $\operatorname{dim}(\ell / \ell \cap g \ell)$ and $\theta^{\ell}(g)$ are just the rank and discriminant of the quadratic form used to define $Q(g \ell, g o ; \ell, o)$ in Section A.5.1. 
Remark 2.6.5. For a brief history of this construction of the metaplectic group and the related calculation of the cocycle of the Weil representation, see the bibliographical note in [Lion and Vergne 1980].

2.7. Intermediate cocycles. The transition from $\mathrm{Mp}(V)$ to $\mathrm{Mp}^{\psi, \ell}(V)$ involves two choices: that of the Lagrangian $\ell \subset V$, and that of the character $\psi$. To clarify the relationship between the different versions of the metaplectic group, we now examine these choices separately.

2.7.1. Choice of Lagrangian. The definitions follow the same pattern as before, and make sense for any $F$.

Definition. Let $M^{\ell}(V)$ be the central extension

$$
0 \rightarrow W(F) / I^{3} \rightarrow M^{\ell}(V) \rightarrow \mathrm{Sp}(V) \rightarrow 1
$$

defined by the cocycle

$$
c_{\ell}(g, h)=\tau(\ell, g \ell, g h \ell) .
$$

Definition [Parimala et al. 2000]. Let $\mathrm{Mp}^{\ell}(V) \subset M^{\ell}(V)$ be the subset of pairs $(g, q)$ such that $q=Q(g \ell, g o ; \ell, o) \bmod I^{2}$ (in the notation of Definition 2.6.3). In other words, $q$ has rank $n:=\operatorname{dim}(\ell / \ell \cap g \ell) \bmod 2$ and signed discriminant $(-1)^{n(n-1) / 2} \theta^{\ell}(g)$ (in the notation of Remark 2.6.4).

With this definition, one can show that $\operatorname{Mp}^{\ell}(V)$ is a subgroup of $M^{\ell}(V)$, and, indeed, it is the unique (cf. Section 2.4.1) subgroup of $M^{\ell}(V)$ yielding a central extension

$$
0 \rightarrow I^{2} / I^{3} \rightarrow \operatorname{Mp}^{\ell}(V) \rightarrow \mathrm{Sp}(V) \rightarrow 1 .
$$

Remark 2.7.2. The following relationship is crucial to the proof of Theorem B. As in Section 2.2 , let $\bar{V}$ be the symplectic vector space $(V,-\omega)$. Then the map $M(V) \rightarrow M^{\Gamma_{1}}(\bar{V} \oplus V)$ given by $(g, q) \mapsto(1 \oplus g, q)$ is a homomorphic embedding (and, by Section 2.4.1, it embeds $\operatorname{Mp}(V)$ into $\operatorname{Mp}^{\Gamma_{1}}(\bar{V} \oplus V)$ ). All of what we have said about $\operatorname{Mp}(V)$ can thereby be reduced to facts about $\operatorname{Mp}^{\Gamma_{1}}(\bar{V} \oplus V)$.

2.7.3. Choice of an additive character. Here we assume that $F$ is finite or local.

Definition. Define a central extension

$$
1 \rightarrow Z_{8} \rightarrow M^{\psi}(V) \rightarrow \mathrm{Sp}(V) \rightarrow 1
$$

using the cocycle

$$
c_{\psi}\left(g, g^{\prime}\right)=\gamma_{\psi}\left(\tau\left(\Gamma_{1}, \Gamma_{g}, \Gamma_{g g^{\prime}}\right)\right) .
$$

We again construct a subgroup $\operatorname{Mp}^{\psi}(V) \subset M^{\psi}(V)$ fitting into a central extension

$$
1 \rightarrow Z_{F} \rightarrow \mathrm{Mp}^{\psi}(V) \rightarrow \mathrm{Sp}(V) \rightarrow 1
$$


and this subgroup is again unique, by Lemma 2.5.

Definition. Let $\operatorname{Mp}^{\psi}(V) \subset M^{\psi}(V)$ be the subgroup consisting of pairs $(g, \xi)$ with $\xi=\gamma_{\psi}\left(\left[\sigma_{g}\right]\right) \bmod Z_{F}$. Equivalently (using Section A.4.1(c)), the requirement is that

$$
\xi=\gamma_{\psi}(1)^{\operatorname{dim}(g-1) V-1} \gamma_{\psi}\left(\operatorname{det} \sigma_{g}\right) \bmod Z_{F} .
$$

\subsection{Remarks.}

Remark 2.8.1. Given the existence of a unique isomorphism $I^{2} / I^{3} \rightarrow Z_{F}$ (Theorem A.2) when $F$ is finite or local, the introduction of a character $\psi$ may seem entirely extraneous to the construction of the metaplectic group. Indeed, its use is motivated by the Weil representation, which may be considered as a representation of $M^{\psi}(V)$ (or $M^{\psi, \ell}(V)$ ) in which the central factor $Z_{8}$ acts by scalar multiplication.

Remark 2.8.2. Let us explain the relationship between the present constructions and the version of the metaplectic group used in [Thomas 2008] (which considered only finite and local fields). Let $\operatorname{Gr}(V)$ be the set of all Lagrangian subspaces $\ell \subset V$. As we explain in the next section, there is a canonical isomorphism $\delta_{\ell \ell^{\prime}}^{\psi}: \operatorname{Mp}^{\psi, \ell}(V) \rightarrow \operatorname{Mp}^{\psi, \ell^{\prime}}(V)$ for every pair $\ell, \ell^{\prime} \in \operatorname{Gr}(V)$. Then

$$
G=\left\{\left(g_{\ell}\right) \in \prod_{\ell \in \operatorname{Gr}(V)} \operatorname{Mp}^{\psi, \ell}(V) \mid \delta_{\ell \ell^{\prime}}^{\psi}\left(g_{\ell}\right)=g_{\ell^{\prime}} \text { for all } \ell, \ell^{\prime} \in \operatorname{Gr}(V)\right\}
$$

is a group under component-wise multiplication, with the obvious projections making $G$ isomorphic to each $\operatorname{Mp}^{\psi, \ell}(V)$. This $G$ is essentially what was called $\mathrm{Mp}(V)$ in [Thomas 2008, Definition 5.2]. By construction, it does not depend on any particular choice of $\ell \in \operatorname{Gr}(V)$; one could, of course, remove the apparent dependence on $\psi$ by a similar trick.

Remark 2.8.3. Suppose that $F$ is a local field. It is well-known that $\operatorname{Mp}^{\psi, \ell}(V)$ is naturally a topological covering group of $\operatorname{Sp}(V)$ - the topology is the one that makes the Weil representation continuous. Since, as explained in the next section, $\operatorname{Mp}(V)$ and $\mathrm{Mp}^{\psi, \ell}(V)$ are canonically isomorphic, this defines a topology on $\operatorname{Mp}(V)$, which can be extended in a unique way to $M(V)$, making $M(V)$ a covering group of $\mathrm{Sp}(V)$ as well. It is interesting to describe this topology more explicitly, by giving an open neighborhood $U$ of the identity $(1,0) \in M(V)$ that maps homeomorphically onto its image in $\operatorname{Sp}(V)$. It turns out we can take

$$
U=\left\{(g, q) \in M(V) \mid \operatorname{ker}(g+1)=0, q=-Q_{g} \bmod I^{3}\right\} .
$$

For example, this means that the formula in Theorem $\mathrm{D}$ is continuous at $(1,0)$. For an analogous description of the topology of $\mathrm{Mp}^{\psi, \ell}(V)$, see [Thomas 2008, Proposition 5.3]. 


\section{Isomorphisms between metaplectic groups}

In this section, we describe isomorphisms between the different versions of the metaplectic group that were introduced in Section 2. First we consider the choice of Lagrangian, describing canonical (see Section 3.1) isomorphisms that fit into a commutative diagram (omitting $V$ from the notation):

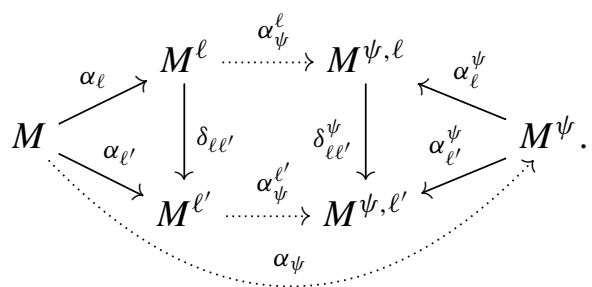

(The dotted arrows are homomorphisms, not isomorphisms, but all the maps shown restrict to isomorphisms between the various groups $\mathrm{Mp}^{\bullet}(V)$.) Next we consider the choice of additive character, describing a commutative diagram of canonical isomorphisms:

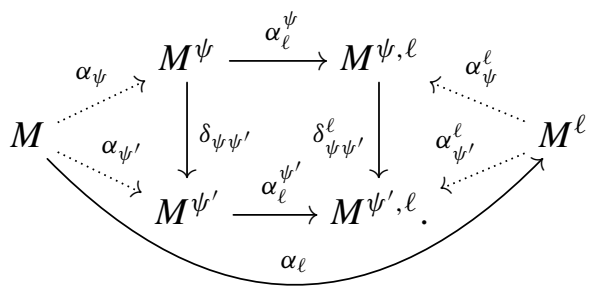

Finally, we describe canonical actions of $\operatorname{GSp}(V)$ on $M(V)$ and $M^{\psi, \ell}(V)$ that cover the action by conjugation on $\operatorname{Sp}(V)$.

As in Section 2, objects labeled by the character $\psi$ are defined only when $F$ is a finite or local field; objects that do not involve $\psi$ make sense more generally.

3.1. In the above overview, we used the word "canonical" to mean "unique" in the following sense. If $\tilde{G}$ and $\tilde{G}^{\prime}$ are central extensions of a group $G$ by an abelian group $A$, then "an isomorphism of central extensions" is an isomorphism $\tilde{G} \rightarrow \tilde{G}^{\prime}$ which covers the identity $G \rightarrow G$ and restricts to the identity $A \rightarrow A$. The claim is that all the isomorphisms are unique as isomorphisms of central extensions. This uniqueness is guaranteed by the following lemma.

Lemma 3.2. Let $\tilde{G}$ and $\tilde{G}^{\prime}$ be central extensions of $\operatorname{Sp}(V)$ by an abelian group A with no 3-torsion. Then there exists at most one isomorphism $\tilde{G} \rightarrow \tilde{G}^{\prime}$ of central extensions.

Proof. If $f_{1}, f_{2}: \tilde{G} \rightarrow \tilde{G}^{\prime}$ are isomorphisms of central extensions, then

$$
(g, a) \mapsto f_{1}(g, a) \cdot f_{2}(g, a)^{-1}
$$


is given by a homomorphism $\operatorname{Sp}(V)=\tilde{G} / A \rightarrow A \subset \tilde{G}^{\prime}$. But, as explained in the proof of Lemma 2.5, any such homomorphism is trivial.

As we noted after Lemma 2.5, the Witt group $W(F)$ has only 2-primary torsion, so Lemma 3.2 applies to all the central extensions of interest.

3.2.1. Coboundary description. We will repeatedly use the following basic observation. If $\tilde{G}$ and $\tilde{G}^{\prime}$ are defined by 2-cocycles $c$ and $c^{\prime}$, then an isomorphism $f: \tilde{G} \rightarrow \tilde{G}^{\prime}$ of central extensions is equivalent to giving a function $s: G \rightarrow A$ such that

$$
c^{\prime}\left(g, g^{\prime}\right)-c\left(g, g^{\prime}\right)=s\left(g g^{\prime}\right)-s(g)-s\left(g^{\prime}\right) .
$$

(This expresses $c^{\prime}-c$ as the coboundary of $s$.) Namely, $f(g, a)=(g, a+s(g))$.

\subsection{Choice of Lagrangian.}

Proposition 3.4. There is a unique isomorphism $\alpha_{\ell}: M(V) \rightarrow M^{\ell}(V)$ of central extensions, and it is given by

$$
\alpha_{\ell}(g, q)=\left(g, q+\tau\left(\ell \oplus \ell, \Gamma_{1}, \Gamma_{g}, \ell \oplus g \ell\right)\right) .
$$

It restricts to an isomorphism $\mathrm{Mp}(V) \rightarrow \mathrm{Mp}^{\ell}(V)$, also unique.

Proof. For $\alpha_{\ell}$ to be an isomorphism, it suffices, by Section 3.2.1, to check

$$
c_{\ell}\left(g, g^{\prime}\right)-c\left(g, g^{\prime}\right)+s(g)+s\left(g^{\prime}\right)-s\left(g g^{\prime}\right)=0
$$

where $s(g):=\tau\left(\ell \oplus \ell, \Gamma_{1}, \Gamma_{g}, \ell \oplus g \ell\right)$. Observe that $\tau(\ell, \ell, \ell)=0$ : according to Section A.5(e), it is represented by the zero bilinear form on $\ell$. Therefore

$$
c_{\ell}\left(g, g^{\prime}\right)=\tau\left(\ell, g \ell, g g^{\prime} \ell\right)=\tau\left(\ell \oplus g \ell, \ell \oplus g g^{\prime} \ell, \ell \oplus \ell\right)
$$

by A.5(c). Moreover, $s\left(g^{\prime}\right)=\tau\left(\ell \oplus g \ell, \Gamma_{g}, \Gamma_{g g^{\prime}}, \ell \oplus g g^{\prime} \ell\right)$ by A.5(d) applied to $(1, g) \in \mathrm{GL}(\bar{V} \oplus V)$. Graphically, then, (17) is a sum over the faces of the polyhedron

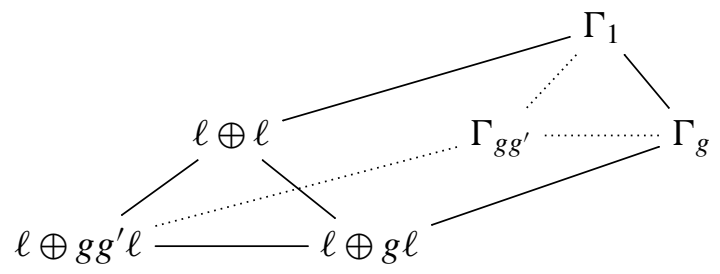

and therefore vanishes, as explained in Section A.5.2.

The fact that $\alpha_{\ell}$ maps $\operatorname{Mp}(V)$ to $\mathrm{Mp}^{\ell}(V)$ follows from the uniqueness property of $\mathrm{Mp}^{\ell}(V)$ (Section 2.4.1), or by direct computation, using (34); the uniqueness of $\alpha_{\ell}$ follows from Lemma 3.2. 
Corollary 3.5. There is a unique isomorphism $\alpha_{\ell}^{\psi}: M^{\psi}(V) \rightarrow M^{\psi, \ell}(V)$ of central extensions, and it is given by

$$
\alpha_{\ell}^{\psi}(g, \xi)=\left(g, \xi \cdot \gamma_{\psi}\left(\tau\left(\ell \oplus \ell, \Gamma_{1}, \Gamma_{g}, \ell \oplus g \ell\right)\right)\right) .
$$

It restricts to an isomorphism $\mathrm{Mp}^{\psi}(V) \rightarrow \mathrm{Mp}^{\psi, \ell}(V)$, also unique.

\subsection{Change of Lagrangian.}

Proposition 3.7. There is a unique isomorphism $\delta_{\ell \ell^{\prime}}: M^{\ell}(V) \rightarrow M^{\ell^{\prime}}(V)$ of central extensions, given by

$$
\delta_{\ell \ell^{\prime}}(g, q)=\left(g, q+\tau\left(\ell, g \ell, g \ell^{\prime}, \ell^{\prime}\right)\right) .
$$

It restricts to an isomorphism $\mathrm{Mp}^{\ell}(V) \rightarrow \mathrm{Mp}^{\ell^{\prime}}(V)$, also unique.

Proof. The proof is very similar to that of Proposition 3.4. The main difference is that we must now show

$$
c_{\ell^{\prime}}\left(g, g^{\prime}\right)-c_{\ell}\left(g, g^{\prime}\right)+s(g)+s\left(g^{\prime}\right)-s\left(g g^{\prime}\right)=0
$$

where now $s(g):=\tau\left(\ell, g \ell, g \ell^{\prime}, \ell^{\prime}\right)$. Observe that $s\left(g^{\prime}\right)=\tau\left(g \ell, g g^{\prime} \ell, g g^{\prime} \ell^{\prime}, g \ell^{\prime}\right)$ by Section A.5(d). Thus (19) is a sum over the faces of the polyhedron

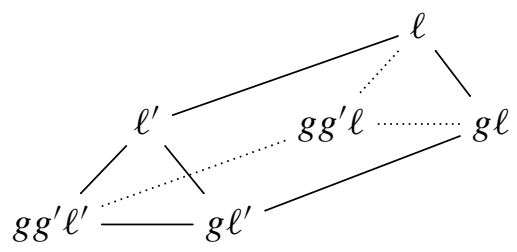

and again vanishes by Section A.5.2.

Corollary 3.8. There is a unique isomorphism $\delta_{\ell \ell^{\prime}}^{\psi}: M^{\psi, \ell}(V) \rightarrow M^{\psi, \ell^{\prime}}(V)$ of central extensions, given by

$$
\delta_{\ell \ell^{\prime}}^{\psi}(g, \xi)=\left(g, \xi \cdot \gamma_{\psi}\left(\tau\left(\ell, g \ell, g \ell^{\prime}, \ell^{\prime}\right)\right)\right) .
$$

It restricts to an isomorphism $\mathrm{Mp}^{\psi, \ell}(V) \rightarrow \mathrm{Mp}^{\psi, \ell^{\prime}}(V)$, also unique.

3.9. Choice of additive character. There are obvious homomorphisms

$$
\alpha_{\psi}: M(V) \rightarrow M^{\psi}(V), \quad \alpha_{\psi}^{\ell}: M^{\ell}(V) \rightarrow M^{\psi, \ell}(V),
$$

each given by $(g, q) \mapsto\left(g, \gamma_{\psi}(q)\right)$.

Proposition 3.10. The maps $\alpha_{\psi}, \alpha_{\psi}^{\ell}$ are the unique homomorphisms that cover the identity on $\operatorname{Sp}(V)$ and restrict to $\gamma_{\psi}: W(F) / I^{3} \rightarrow Z_{8}$. Moreover, they restrict to isomorphisms

$$
\alpha_{\psi}: \operatorname{Mp}(V) \rightarrow \operatorname{Mp}^{\psi}(V), \quad \alpha_{\psi}^{\ell}: \operatorname{Mp}^{\ell}(V) \rightarrow \operatorname{Mp}^{\psi, \ell}(V),
$$

that are unique as isomorphisms of central extensions. 
Proof. Uniqueness is a simple variation on Lemma 3.2. The fact that $\operatorname{Mp}(V)$ and $\operatorname{Mp}^{\ell}(V)$ map to $\operatorname{Mp}^{\psi}(V)$ and $\operatorname{Mp}^{\psi, \ell}(V)$ is immediate from the definitions. The fact that the restricted maps are isomorphisms follows from the fact that

$$
\gamma_{\psi}: I^{2} / I^{3} \rightarrow Z_{F}
$$

is an isomorphism (Section A.4.1(d)).

3.11. Change of additive character. Suppose that $\psi, \psi^{\prime}$ are nontrivial additive characters of $F$. Let $a \in F^{\times}$be the unique scalar such that $\psi^{\prime}(x)=\psi(a x)$ for all $x \in F$. In the next proposition, $(\cdot, \cdot)_{H}: F^{\times} \otimes_{\mathbb{Z}} F^{\times} \rightarrow Z_{F}$ is the Hilbert symbol (defined in Section A.1.2).

Proposition 3.12. There is a unique isomorphism $\delta_{\psi \psi^{\prime}}: M^{\psi}(V) \rightarrow M^{\psi^{\prime}}(V)$ of central extensions, and it is given by

$$
\delta_{\psi \psi^{\prime}}(g, \xi)=\left(g, r_{a}(g) \xi\right)
$$

where $r_{a}(g):=\left(\gamma_{\psi}(a) / \gamma_{\psi}(1)\right)^{\operatorname{dim}(g-1) V}\left(a, \operatorname{det} \sigma_{g}\right)_{H}$. It restricts to an isomorphism $\mathrm{Mp}^{\psi}(V) \rightarrow \mathrm{Mp}^{\psi^{\prime}}(V)$, also unique.

To prove Proposition 3.12, we first study the dependence of $\gamma_{\psi}$ on $\psi$.

Lemma 3.13. For any quadratic space $(A, q)$,

$$
\gamma_{\psi^{\prime}}(q)=\gamma_{\psi}(q)\left(\gamma_{\psi}(a) / \gamma_{\psi}(1)\right)^{\operatorname{dim} A}(a, \operatorname{det} q)_{H} .
$$

Proof. Both sides of the equation define homomorphisms $W(F) \rightarrow \mathbb{C}^{\times}$. Since any quadratic space is the perpendicular sum of one-dimensional ones, we can reduce to the case where $A=F$ and $q(x, y)=b x y$. Then $\gamma_{\psi^{\prime}}(q)=\gamma_{\psi}(a b)$ and the statement amounts to the standard formula Section A.4.1(b).

Proof of Proposition 3.12. To get an isomorphism, by Section 3.2.1 we must check

$$
\gamma_{\psi^{\prime}}\left(\tau\left(\Gamma_{1}, \Gamma_{g}, \Gamma_{g g^{\prime}}\right)\right)=\gamma_{\psi}\left(\tau\left(\Gamma_{1}, \Gamma_{g}, \Gamma_{g g^{\prime}}\right)\right) \cdot \frac{r_{a}\left(g g^{\prime}\right)}{r_{a}(g) r_{a}\left(g^{\prime}\right)} .
$$

The right-hand side simplifies to

$$
\gamma_{\psi}\left(\tau\left(\Gamma_{1}, \Gamma_{g}, \Gamma_{g g^{\prime}}\right)\right) \cdot\left(\gamma_{\psi}(a) / \gamma_{\psi}(1)\right)^{d}(a, \delta)_{H},
$$

where

$d=\operatorname{dim}\left(g g^{\prime}-1\right) V-\operatorname{dim}(g-1) V-\operatorname{dim}\left(g^{\prime}-1\right) V$ and $\delta=\operatorname{det} \sigma_{g g^{\prime}} /\left(\operatorname{det} \sigma_{g} \operatorname{det} \sigma_{g^{\prime}}\right)$.

Comparing this to Lemma 3.13, we are reduced to checking that $\tau\left(\Gamma_{1}, \Gamma_{g}, \Gamma_{g g^{\prime}}\right)$ has rank $d \bmod 2$ and signed discriminant $(-1)^{d(d-1) / 2} \delta$. This is equivalent to (9).

We therefore have an isomorphism; uniqueness follows from Lemma 3.2, and the fact that $\mathrm{Mp}^{\psi}(V)$ maps to $\mathrm{Mp}^{\psi^{\prime}}(V)$ follows from Lemma 2.5.

Here is the analogue of Proposition 3.12 for $M^{\psi, \ell}(V)$. 
Proposition 3.14. There is a unique isomorphism $\delta_{\psi \psi^{\prime}}^{\ell}: M^{\psi, \ell}(V) \rightarrow M^{\psi^{\prime}, \ell}(V)$ of central extensions, and it is given by

$$
\delta_{\psi \psi^{\prime}}^{\ell}(g, \xi)=\left(g, r_{a}^{\ell}(g) \xi\right)
$$

where $r_{a}^{\ell}(g):=\left(\gamma_{\psi}(a) / \gamma_{\psi}(1)\right)^{\operatorname{dim}(\ell / \ell \cap g \ell)}\left(a, \theta^{\ell}(g)\right)_{H}$. It restricts to an isomorphism $\operatorname{Mp}^{\psi, \ell}(V) \rightarrow \operatorname{Mp}^{\psi^{\prime}, \ell}(V)$, also unique.

3.15. Outer automorphisms. We return to the general setting where $F$ is any field of characteristic not 2. Let $\mathrm{GSp}(V) \subset \mathrm{GL}(V)$ be the group of symplectic similitudes, that is, linear transformations $f \in \mathrm{GL}(V)$ such that there exists $\lambda(f) \in F^{\times}$satisfying $\omega(f x, f y)=\lambda(f) \omega(x, y)$ for all $x, y \in V$. Then $\operatorname{GSp}(V)$ contains $\operatorname{Sp}(V)$ as a normal subgroup, and so acts on it by conjugation. (In fact, according to [Hua 1948], any automorphism of $\operatorname{Sp}(V)$ can be written as a composition $\varphi \circ \operatorname{Ad} f$ with $f \in \mathrm{GSp}(V)$ and $\varphi$ a field automorphism of $F$.)

The goal of this section is to describe explicitly an action of $\operatorname{GSp}(V)$ on the metaplectic group, lifting the conjugation action on $\operatorname{Sp}(V)$. This lifting is unique.

First let us define a function

$$
\mathrm{Sp}(V) \times F^{\times} \rightarrow W(F) / I^{3} .
$$

Given $(g, a) \in \mathrm{Sp}(V) \times F^{\times}$, let $b_{g} \in W(F)$ be represented by a quadratic space of rank $\operatorname{dim}(g-1) V$ and discriminant $\operatorname{det} \sigma_{g}$ (thus $b_{g}=\left[\sigma_{g}\right]$ modulo $I^{2}$ ). Now let $q_{g, a}=\left(q_{a}-1\right) \otimes b_{g}$. The class of $q_{g, a}$ in $W(F) / I^{3}$ is independent of choices.

Proposition 3.16. For any $f \in \mathrm{GSp}(V)$ there is a unique automorphism $N_{f}$ of $M(V)$ covering $\operatorname{Ad} f$ and restricting to the identity on $W(F) / I^{3}$. It is given by $N_{f}(g, q)=\left(\operatorname{Ad} f(g), q+q_{g, \lambda(f)}\right)$.

Proof. Simple variations on Lemma 3.2 and Section 3.2.1 show that $N_{f}$ will be a unique isomorphism so long as

$$
\tau\left(\Gamma_{1}, \Gamma_{\mathrm{Ad} f(g)}, \Gamma_{\mathrm{Ad} f\left(g g^{\prime}\right)}\right)-\tau\left(\Gamma_{1}, \Gamma_{g}, \Gamma_{g g^{\prime}}\right)=q_{g g^{\prime}, \lambda(f)}-q_{g, \lambda(f)}-q_{g^{\prime}, \lambda(f)}
$$

modulo $I^{3}$. Now,

$$
\Gamma_{\text {Ad } f(g)}=\left\{\left(v, f g f^{-1} v\right)\right\}=\{(f v, f g v)\}=(f, f) \cdot \Gamma_{g} \subset \bar{V} \oplus V .
$$

This and Section A.5(d) imply that

$$
\tau\left(\Gamma_{1}, \Gamma_{\mathrm{Ad} f(g)}, \Gamma_{\mathrm{Ad} f\left(g g^{\prime}\right)}\right)=q_{\lambda(f)} \otimes \tau\left(\Gamma_{1}, \Gamma_{g}, \Gamma_{g g^{\prime}}\right) .
$$

Thus the left-hand side of $(20)$ is $\left(q_{\lambda(f)}-1\right) \otimes \tau\left(\Gamma_{1}, \Gamma_{g}, \Gamma_{g g^{\prime}}\right)$. By the definition of $q_{g, \lambda(f)}$, to establish (20), it suffices to show that

$$
\tau\left(\Gamma_{1}, \Gamma_{g}, \Gamma_{g g^{\prime}}\right)=b_{g g^{\prime}}-b_{g}-b_{g^{\prime}} \bmod I^{2} .
$$

But this is equivalent to (9). 
Remark 3.16.1. Proposition 3.16 is stated for $M(V)$, but the uniqueness of $\operatorname{Mp}(V)$ (Section 2.4.1) implies that $N_{f}$ restricts to an automorphism of that subgroup, which is again the unique automorphism covering $\operatorname{Ad} f$.

A description of the automorphisms of $M^{\ell}(V), M^{\psi}(V)$, and $M^{\psi, \ell}(V)$ covering the action of $\operatorname{GSp}(V)$ is easily deduced in parallel to Proposition 3.16, using the isomorphisms of Sections 3.6-3.11. For example, we have:

Proposition 3.17. For any $f \in \mathrm{GSp}(V)$ there is a unique automorphism $N_{f}^{\psi, \ell}$ of $M^{\psi, \ell}(V)$ covering $\operatorname{Ad} f$ and restricting to the identity on $Z_{8}$. It is given by

$$
N_{f}^{\psi, \ell}(g, \xi)=\left(\operatorname{Ad} f(g), \gamma_{\psi}\left(\tau\left(\ell, g \ell, g f^{-1} \ell, f^{-1} \ell\right)\right) \cdot r_{\lambda(f)}^{f^{-1} \ell}(g) \cdot \xi\right) .
$$

Proof. Put $a:=\lambda(f), \psi^{\prime}(x)=\psi(a x)$, and $\ell^{\prime}=f^{-1} \ell$. By Section A.5(d), we have

$$
\begin{aligned}
c_{\psi, \ell}\left(\operatorname{Ad} f(g), \operatorname{Ad} f\left(g^{\prime}\right)\right) & =\gamma_{\psi}\left(\tau\left(\ell, f g f^{-1} \ell, f g g^{\prime} f^{-1} \ell\right)\right. \\
& =\gamma_{\psi^{\prime}}\left(\tau\left(\ell^{\prime}, g \ell^{\prime}, g g^{\prime} \ell^{\prime}\right)\right)=c_{\psi^{\prime}, \ell^{\prime}}\left(g, g^{\prime}\right) .
\end{aligned}
$$

It follows that $s:(g, \xi) \mapsto(\operatorname{Ad} f(g), \xi)$ is an isomorphism $M^{\psi^{\prime}, \ell^{\prime}}(V) \rightarrow M^{\psi, \ell}(V)$ and thence that $N_{f}^{\psi, \ell}(g, \xi)=s \circ \delta_{\psi \psi^{\prime}}^{\ell^{\prime}} \circ \delta_{\ell \ell^{\prime}}^{\psi}$ is an automorphism of $M^{\psi, \ell}(V)$ of the required kind.

Remark 3.17.1. Proposition 3.17 is related to Proposition 3.16 in the sense that we must have $N_{f}^{\psi, \ell} \circ \alpha_{\psi}^{\ell} \circ \alpha_{\ell}=\alpha_{\psi}^{\ell} \circ \alpha_{\ell} \circ N_{f}$. (One can even use this to deduce Proposition 3.17 from Proposition 3.16, but the proof we have presented is much easier, given what we already know.)

\section{Heisenberg group and Weil representation}

Henceforth $F$ is a finite or local field with characteristic not 2 .

In this section we recall the definition and basic properties of the Weil representation $\rho^{\psi, \ell}$. A more detailed exposition can be found in [Lion and Vergne 1980, $\S 1.2-1.4$ and Appendix].

4.1. Hilbert spaces and norms. In describing representations, we use natural Hilbert spaces of half-densities, with the notation laid out in Section A.3.1. Thus if $X$ is a finite-dimensional vector space over $F$ then $L^{2}(X)$ denotes the space of $L^{2}$ functions $X \rightarrow \Omega_{1 / 2}(X)$.

4.2. The Heisenberg group. The Heisenberg group $H(V)$ based on $V$ is, as a set, the direct product $H(V)=V \times F$, equipped with the multiplication

$$
(v, s)(w, t)=\left(v+w, s+t+\frac{1}{2} \omega(v, w)\right) .
$$

The center of $H(V)$ is the factor $F$. We are interested in representations of $H(V)$ with fixed central character $\psi$ (so again $\psi$ is a continuous homomorphism 
$F \rightarrow U(1))$. To avoid always writing the action of the center, note that such a representation $\rho$ is determined by the family of operators $\{\rho(v)\}_{v \in V}$, which satisfy

$$
\rho(v) \rho(w)=\psi\left(\frac{1}{2} \omega(v, w)\right) \cdot \rho(v+w) .
$$

Theorem 4.3 (Stone and von Neumann). $H(V)$ has, for each nontrivial central character $\psi$, a unique isomorphism class of unitary, continuous, irreducible representations. (The notion of continuity is that of the strong operator topology.)

The proof over $\mathbb{R}$ can be found in [Lion and Vergne 1980, §1.3], and a general exposition is in [Prasad 2011]. The main step is Proposition 5.2(a) below.

4.4. Formulas for its representation. For chosen $\ell \in \operatorname{Lagr}(V)$, the representation from Theorem 4.3 is realized by

$$
\rho_{H}^{\psi, \ell}:=\operatorname{Ind}_{\ell \times F}^{H}(\tilde{\psi})
$$

where $\tilde{\psi}$ is the composition $\ell \times F \rightarrow F \stackrel{\psi}{\rightarrow} \mathbb{C}^{\times}$. One has the following explicit description of the corresponding Hilbert space $\mathscr{H}^{\psi, \ell}$. It is the completion of the space of smooth functions $\phi: V \rightarrow \Omega_{1 / 2}(V / \ell)$ that satisfy

$$
\phi(v+w)=\phi(v) \psi\left(\frac{1}{2} \omega(v, w)\right) \text { for all } w \in \ell
$$

and that are finite under the norm

$$
|\phi|^{2}:=\int_{v \in V / \ell} \overline{\phi(v)} \phi(v) .
$$

The action of $H(V)$ on $\mathscr{H}^{\psi, \ell}$ is given, for $\phi \in \mathscr{H}^{\psi, \ell}$ and $v \in V$, by

$$
\rho_{H}^{\psi, \ell}(v) \phi(x)=\phi(x-v) \psi\left(\frac{1}{2} \omega(v, x)\right) .
$$

4.4.1. Transverse Lagrangians. For any Lagrangian $\ell^{\prime}$ transverse to $\ell$, the isomorphism $V / \ell \rightarrow \ell^{\prime}$ yields an isometry

$$
\operatorname{Res}_{\ell^{\prime}}: \mathscr{H}^{\psi, \ell} \rightarrow L^{2}\left(\ell^{\prime}\right)
$$

The action of $H(V)$ on $L^{2}\left(\ell^{\prime}\right)$ is described by the formula

$$
\left(\operatorname{Res}_{\ell^{\prime}} \circ \rho_{H}^{\psi, \ell}\left(v+v^{\prime}\right) \circ \operatorname{Res}_{\ell^{\prime}}^{-1}\right)(\phi)\left(x^{\prime}\right)=\phi\left(x^{\prime}-v^{\prime}\right) \cdot \psi\left(\omega\left(v, x^{\prime}-\frac{1}{2} v^{\prime}\right)\right)
$$

for all $v \in \ell$ and $v^{\prime}, x^{\prime} \in \ell^{\prime}$.

4.5. The Weil representation. Since $\operatorname{Sp}(V)$ is the group of automorphisms of $H(V)$ preserving the center, one obtains a projective representation $\rho_{\mathrm{Sp}}^{\psi, \ell}$ of $\operatorname{Sp}(V)$ acting on $\mathscr{H}^{\psi, \ell}$, characterized by

$$
\rho_{\mathrm{Sp}}^{\psi, \ell}(g) \circ \rho_{H}^{\psi, \ell}(v) \circ \rho_{\mathrm{Sp}}^{\psi, \ell}(g)^{-1}=\rho_{H}^{\psi, \ell}(g v) .
$$


In detail, $\left(\rho_{H}^{\psi, \ell}\right)^{g}: v \mapsto \rho_{H}^{\psi, \ell}(g v)$ defines a representation of $H(V)$ on $\mathscr{H}^{\psi, \ell}$ with central character $\psi$. By Theorem 4.3 , there is a unique-up-to-scale operator $\rho_{\mathrm{Sp}}^{\psi, \ell}(g)$ on $\mathscr{H}^{\psi, \ell}$ intertwining from $\rho_{H}^{\psi, \ell}$ to $\left(\rho_{H}^{\psi, \ell}\right)^{g}$.

The next result is due to Lion and Perrin.

Theorem 4.6 [Perrin 1981]. There is a true representation $\rho_{\mathrm{Mp}}^{\psi, \ell}$ of $\mathrm{Mp}^{\psi, \ell}(V)$, uniquely characterized by the formulas

$$
\rho_{\mathrm{Mp}}^{\psi, \ell}(g, \xi) \circ \rho_{H}^{\psi, \ell}(v) \circ \rho_{\mathrm{Mp}}^{\psi, \ell}(g, \xi)^{-1}=\rho_{H}^{\psi, \ell}(g v), \quad \rho_{\mathrm{Mp}}^{\psi, \ell}(1, \xi)=\xi \cdot \mathrm{id} .
$$

The operators $\rho_{\mathrm{Mp}}^{\psi, \ell}(g, \xi): \mathscr{H}^{\psi, \ell} \rightarrow \mathscr{H}^{\psi, \ell}$ are given on Schwartz functions $\phi$ by

$$
\rho_{\mathrm{Mp}}^{\psi, \ell}(g, \xi) \phi(x):=\xi \cdot \int_{y \in\left(g^{-1} \ell\right) /\left(\ell \cap g^{-1} \ell\right)} \phi\left(g^{-1} x+y\right) \psi\left(\frac{1}{2} \omega\left(y, g^{-1} x\right)\right) \mu_{g}^{\psi, \ell}
$$

where $\mu_{g}^{\psi, \ell} \in \Omega_{1}\left(\left(g^{-1} \ell\right) /\left(\ell \cap g^{-1} \ell\right)\right)$ is the unique invariant measure such that $\rho_{\mathrm{Mp}}^{\psi, \ell}(g, \xi)$ is unitary.

Remark 4.6.1. More concretely, $\mu_{g}^{\psi, \ell}$ is characterized by the following property. First, $g^{-1} \ell /\left(\ell \cap g^{-1} \ell\right)$ and $\ell /\left(\ell \cap g^{-1} \ell\right)$ are Pontryagin-dual abelian groups under the pairing $\psi \circ \omega$. Let $\mu$ be the measure on $\ell /\left(\ell \cap g^{-1} \ell\right)$ dual to $\mu_{g}^{\psi, \ell}$. Choose a measure $\mu_{0}$ on $\ell \cap g^{-1} \ell$. Then $\mu_{g}^{\psi, \ell} \otimes \mu_{0}$ and $\mu \otimes \mu_{0}$ are measures on $g^{-1} \ell$ and $\ell$, respectively. The property is that these measures correspond under the isomorphism $g: g^{-1} \ell \rightarrow \ell$.

4.7. Definition. Let $\rho^{\psi, \ell}$ be the representation of $\mathrm{Mp}^{\psi, \ell}(V) \ltimes H(V)$ defined by

$$
\rho^{\psi, \ell}(g, \xi ; v, t)=\rho_{\mathrm{Mp}}^{\psi, \ell}(g, \xi) \circ \rho_{H}^{\psi, \ell}(v, t) .
$$

We also use $\rho^{\psi, \ell}$ to denote the corresponding representation of $\operatorname{Mp}(V) \ltimes H(V)$, defined using the canonical isomorphism $\alpha_{\psi}^{\ell} \circ \alpha_{\ell}=\alpha_{\ell}^{\psi} \circ \alpha_{\psi}: \operatorname{Mp}(V) \rightarrow \operatorname{Mp}^{\psi, \ell}(V)$. Thus for $q \in W(F) / I^{3}$,

$$
\rho_{\mathrm{Mp}}^{\psi, \ell}(g, q):=\rho_{\mathrm{Mp}}^{\psi, \ell}(g, \xi), \quad \text { with } \xi:=\gamma_{\psi}\left(q+\tau\left(\ell \oplus \ell, \Gamma_{1}, \Gamma_{g}, \ell \oplus g \ell\right)\right) \in Z_{8} .
$$

\section{The character: Proof of Theorem B}

The goal of this section is to prove Theorem B. There are two main ideas involved: first, the Weyl transform, developed in Section 5.1, and second, the homomorphism $\operatorname{Sp}(V) \rightarrow \operatorname{Sp}(\bar{V} \oplus V)$, studied in Section 5.4. We conclude the proof of Theorem B in Section 5.6. 
5.1. Weyl transform. Let $\mathscr{Y}(V) \subset L^{2}(V)$ be the subspace of Schwartz-class halfdensities. ${ }^{6}$ Let $\operatorname{End}_{0} \mathscr{H}^{\psi, \ell} \cong \mathscr{S}(V / \ell \times V / \ell)$ be the algebra of operators on the Hilbert space $\mathscr{H}^{\psi, \ell} \cong L^{2}(V / \ell)$ that can be represented by Schwartz-class integral kernels. It is dense in the algebra End $\mathscr{H}^{\psi, \ell} \cong L^{2}(V / \ell \times V / \ell)$ of Hilbert-Schmidt operators (that is, those with $L^{2}$ integral kernels).

The following proposition is well-known (it is the heart of the Stone-von Neumann Theorem, 4.3). As usual, $\mu_{V}$ denotes the measure on $V$ self-dual with respect to $\psi \circ \omega$.

Proposition 5.2. For $h \in \mathscr{Y}(V)$, let $W^{\psi, \ell}(h)$ be the operator on $\mathscr{H}^{\psi, \ell}$ defined by

$$
W^{\psi, \ell}(h)(\phi)(x)=\int_{v \in V} \rho_{H}^{\psi, \ell}(v) \phi(x) \cdot h(v) \mu_{V}^{1 / 2} .
$$

(a) $W^{\psi, \ell}$ is an isomorphism $\mathscr{Y}(V) \rightarrow \operatorname{End}_{0}\left(\mathscr{H}^{\psi, \ell}\right)$ and extends to an isometry

$$
W^{\psi, \ell}: L^{2}(V) \rightarrow \operatorname{End}\left(\mathscr{H}^{\psi, \ell}\right) .
$$

(b) If we equip $L^{2}(V)$ with the multiplication

$$
\left(f_{1} \star f_{2}\right)(x):=\int_{v \in V} f_{1}(v) \psi\left(\frac{1}{2} \omega(v, x)\right) f_{2}(x-v) \mu_{V}^{1 / 2}
$$

then $W^{\psi, \ell}$ becomes an algebra isomorphism $W^{\psi, \ell}: L^{2}(V) \rightarrow \operatorname{End}\left(\mathscr{H}^{\psi, \ell}\right)$.

(c) For $h \in \mathscr{Y}(V)$, the operator $W^{\psi, \ell}(h)$ is trace class, and

$$
\operatorname{Tr} W^{\psi, \ell}(h) \cdot \mu_{V}^{1 / 2}=h(0) .
$$

Proof. Choose $\ell^{\prime}$ transverse to $\ell$, and identify $L^{2}(V)=L^{2}\left(\ell \times \ell^{\prime}\right)$. Let $\mathfrak{F}_{0}$ be the Fourier transform $L^{2}(\ell) \rightarrow L^{2}\left(\ell^{\prime}\right)$ with respect to the pairing $\psi \circ \frac{1}{2} \omega$ :

$$
\mathfrak{F}_{0} f\left(a^{\prime}\right):=\|2\|^{-\frac{\operatorname{dim} V}{4}} \int_{a \in \ell} f(a) \psi\left(\frac{1}{2} \omega\left(a, a^{\prime}\right)\right) \mu_{V}^{1 / 2} .
$$

(There is a canonical isomorphism $\Omega_{1 / 2}(\ell) \otimes \Omega_{1 / 2}(V)=\Omega_{1}(\ell) \otimes \Omega_{1 / 2}\left(\ell^{\prime}\right)$ which allows us to interpret $\mathfrak{F}_{0}$ as a map from half-densities on $\ell$ to half-densities on $\ell^{\prime}$.) Let $A \in \mathrm{GL}\left(\ell^{\prime} \times \ell^{\prime}\right)$ be the isomorphism $A\left(a^{\prime}, x^{\prime}\right)=\left(x^{\prime}+a^{\prime}, x^{\prime}-a^{\prime}\right)$. Write $A^{*}$ for the corresponding isometry $f \mapsto\|2\|^{(\operatorname{dim} V) / 4}(f \circ A)$ of $L^{2}\left(\ell \times \ell^{\prime}\right)$.

Lemma 5.3. $W^{\psi, \ell}$ factors as a composition of isometries

$$
L^{2}(V)=L^{2}\left(\ell \times \ell^{\prime}\right) \stackrel{\mathfrak{F}_{0} \otimes \mathrm{id}}{\longrightarrow} L^{2}\left(\ell^{\prime} \times \ell^{\prime}\right) \stackrel{A^{*}}{\longrightarrow} L^{2}\left(\ell^{\prime} \times \ell^{\prime}\right)=\operatorname{End}\left(\mathscr{H}^{\psi, \ell}\right) .
$$

\footnotetext{
${ }^{6}$ Our exposition here differs slightly from the sketch in Section 1.3(C) in that we use half-densities rather than complex-valued functions; the square root $\mu_{V}^{1 / 2}$ of the self-dual measure for $\psi \circ \omega$ can be used to pass between the two.
} 
Proof. By (25) and (24), we have

$$
\begin{aligned}
W^{\psi, \ell}(h) \phi\left(x^{\prime}\right) & =\int_{\left(a, a^{\prime}\right) \in V} \phi\left(x^{\prime}-a^{\prime}\right) \cdot \psi\left(\omega\left(a, x^{\prime}-\frac{1}{2} a^{\prime}\right)\right) \cdot h\left(a, a^{\prime}\right) \mu_{V}^{1 / 2} \\
& =\int_{a^{\prime} \in \ell^{\prime}} \phi\left(a^{\prime}\right) \int_{a \in \ell} \psi\left(\frac{1}{2} \omega\left(a, x^{\prime}+a^{\prime}\right)\right) \cdot h\left(a, x^{\prime}-a^{\prime}\right) \mu_{V}^{1 / 2}
\end{aligned}
$$

with a change of variables $a^{\prime} \mapsto x^{\prime}-a^{\prime}$; this is exactly what the lemma claims.

Part (a) of the proposition follows from the fact that Fourier transforms preserve the Schwartz class. In part (b), the $\star$-product is just the product induced on $L^{2}(V)$ by viewing it as the $\psi$-coinvariants of the group algebra $L^{2}(H(V))$; thus the fact that $W^{\psi, \ell}$ is a homomorphism is just due to the fact that $\rho_{H}^{\psi, \ell}$ is a representation.

As for part (c), formula (26) expresses $W^{\psi, \ell}(h)$ as a smooth integral kernel; we calculate the trace by integrating along the diagonal $x^{\prime}=a^{\prime}$ to find

$$
\operatorname{Tr} W^{\psi, \ell}(h) \cdot \mu_{V}^{1 / 2}=\int_{a^{\prime} \in \ell^{\prime}} \int_{a \in \ell} \psi\left(\omega\left(a, a^{\prime}\right)\right) \cdot h(a, 0) \mu_{V}=h(0),
$$

the last equality being Fourier inversion.

Remark 5.3.1. Since trace-class operators form an ideal among bounded operators, we conclude from Proposition 5.2(c) that for any $(g, q) \in \operatorname{Mp}(V)$ and any $h$ smooth and compactly supported (or even Schwartz) on $V$, the composed operator $\rho_{\mathrm{Mp}}^{\psi, \ell}(g, q) \circ W^{\psi, \ell}(h)$ is also trace-class; its trace is the integral of $T_{(g, q)}^{\psi}$ against $h$ (this is the defining property of $T_{(g, q)}^{\psi}$ in Theorem B). Moreover, if $h$ is now compactly supported on $\operatorname{Mp}^{\psi, \ell}(V) \ltimes H(V)$, we can see why $\operatorname{Tr} \rho^{\psi, \ell}(h)$ - that is, the right-hand side of (2) - is well-defined. For let $h_{g, \xi, t}$ be the restriction of $h$ to

$$
\{(g, \xi)\} \times V \times\{t\} \subset \mathrm{Mp}^{\psi, \ell}(V) \ltimes H(V) .
$$

Then $(g, \xi, t) \mapsto \psi(t) \rho_{\mathrm{Mp}}^{\psi, \ell}(g, \xi) \circ W^{\psi, \ell}\left(h_{g, \xi, t}\right)$ is a continuous, compactly supported, hence integrable function from $\mathrm{Mp}^{\psi, \ell}(V) \times F$ to trace-class operators, and the trace of its integral is $\operatorname{Tr} \rho^{\psi, \ell}(h)$.

5.4. Doubling. The metaplectic group $\mathrm{Mp}(V)$ acts on $L^{2}(V)$ in two ways. First we have a representation $A_{1}$,

$$
A_{1}(g, q)(h):=\left(W^{\psi, \ell}\right)^{-1}\left(\rho_{\mathrm{Mp}}^{\psi, \ell}(g, q) \circ W^{\psi, \ell}(h)\right) .
$$

(The right-hand side makes sense $-\rho_{\mathrm{Mp}}^{\psi, \ell}(g, q) \circ W^{\psi, \ell}(h)$ is in the image of $W^{\psi, \ell}-$ because Hilbert-Schmidt operators form an ideal.) An integral formula for $A_{1}$ will be given in Proposition 6.2. Second, let us identify $\operatorname{Sp}(V)$ with the subgroup of 
$\operatorname{Sp}(\bar{V} \oplus V)$ acting trivially on $\bar{V}$. The subgroup of $\operatorname{Mp}^{\Gamma_{1}}(\bar{V} \oplus V)$ over $\operatorname{Sp}(V)$ is precisely $\operatorname{Mp}(V)$ (see Remark 2.7.2). We have an isomorphism

$$
b: V \rightarrow \Gamma_{-1}, \quad b(x)=(-x / 2, x / 2)
$$

and the restriction map $\operatorname{Res}_{\Gamma_{-1}}: \mathscr{H}^{\psi, \Gamma_{1}} \rightarrow L^{2}\left(\Gamma_{-1}\right)$ as in Section 4.4.1. Define

$$
R: \mathscr{H}^{\psi, \Gamma_{1}} \rightarrow L^{2}(V), \quad R:=b^{*} \circ \operatorname{Res}_{\Gamma_{-1}}
$$

so that $\mathrm{Mp}(V)$ acts on $L^{2}(V)$ by $A_{2}(g, q):=R \circ \rho_{\mathrm{Mp}}^{\psi, \Gamma_{1}}(g, q) \circ R^{-1}$.

Proposition 5.5. $A_{1}=A_{2}$.

Proof. Consider the representations $B_{1}, B_{2}$ of $H(\bar{V} \oplus V)$ on $L^{2}(V)$ defined by

$$
\begin{aligned}
& B_{1}(\bar{v}, v) h(x)=\left(W^{\psi, \ell}\right)^{-1}\left(\rho_{H}^{\psi, \ell}(v) \circ W^{\psi, \ell}(h) \circ \rho_{H}^{\psi, \ell}(\bar{v})^{-1}\right) \\
& B_{2}(\bar{v}, v) h(x)=R \circ \rho_{H}^{\psi, \Gamma_{1}}(\bar{v}, v) \circ R^{-1}(h)(x)
\end{aligned}
$$

for all $(\bar{v}, v) \in \bar{V} \oplus V$. We have

$$
A_{i}(g, q) \circ B_{i}(\bar{v}, v) \circ A_{i}(g, q)^{-1}=B_{i}(\bar{v}, g v), \quad B_{i}(1, q)=\gamma_{\psi}(q) \cdot \mathrm{id}
$$

for $i=1,2$, and, as in Theorem 4.6, $A_{2}$ is uniquely characterized by these equations. We show that in fact $B_{1}=B_{2}$, from which it follows that $A_{1}=A_{2}$.

Write $b^{\prime}(v):=(v / 2, v / 2)$ for $v \in V$, so that $(\bar{v}, v)=b(v-\bar{v})+b^{\prime}(v+\bar{v})$. Then

$$
\begin{aligned}
B_{2}(\bar{v}, v) h(x) & =\left(R \circ \rho_{H}^{\psi, \Gamma_{1}}(\bar{v}, v) \circ R^{-1}\right)(h)(x) \\
& =\left(\rho_{H}^{\psi, \Gamma_{1}}\left(b(v-\bar{v})+b^{\prime}(v+\bar{v})\right) \circ R^{-1}\right)(h)(b(x)) \\
& =\left(R^{-1}(h)\right)(b(x)-b(v-\bar{v})) \cdot \psi\left(\omega\left(b^{\prime}(v+\bar{v}), b(x-(v-\bar{v}) / 2)\right)\right) \\
& =h(x+\bar{v}-v) \cdot \psi\left(\frac{1}{2} \omega(v+\bar{v}, x+\bar{v})\right)
\end{aligned}
$$

using (24) for the third equality. On the other hand,

$\rho_{H}^{\psi, \ell}(v) \circ W^{\psi, \ell}(h) \circ \rho_{H}^{\psi, \ell}(\bar{v})^{-1}$

$$
\begin{aligned}
& =\int_{x \in V} h(x) \rho_{H}^{\psi, \ell}(v) \rho_{H}^{\psi, \ell}(x) \rho_{H}^{\psi, \ell}(\bar{v})^{-1} \mu_{V}^{1 / 2} \\
& =\int_{x \in V} h(x) \psi\left(\frac{1}{2} \omega(v+\bar{v}, x+v)\right) \rho_{H}^{\psi, \ell}(v+x-\bar{v}) \mu_{V}^{1 / 2} \\
& =\int_{x \in V} h(x+\bar{v}-v) \psi\left(\frac{1}{2} \omega(v+\bar{v}, x+\bar{v})\right) \rho_{H}^{\psi, \ell}(x) \mu_{V}^{1 / 2}
\end{aligned}
$$

using the multiplication law of $H(V)$ and then a change of variables. It follows that $B_{1}(v) h(x)=h(x+\bar{v}-v) \cdot \psi\left(\frac{1}{2} \omega(v+\bar{v}, x+\bar{v})\right)=B_{2}(v) h(x)$ as claimed. 
5.6. Proof of Theorem B. By the definition of $T_{(g, q)}^{\psi}$, we have

$$
\int_{V} T_{(g, q)}^{\psi} h \mu_{V}^{1 / 2}=\operatorname{Tr}\left(\rho_{\mathrm{Mp}}^{\psi, \ell}(g, q) \circ W^{\psi, \ell}(h)\right) \mu_{V}^{1 / 2}
$$

for any $h \in \mathscr{S}(V)$. According to Proposition 5.2(c), the right-hand side equals $A_{1}(g, q) h(0)$. Therefore, by Proposition 5.5 and Theorem 4.6, we have

$$
\begin{aligned}
\int_{V} T_{(g, q)}^{\psi} h \mu_{V}^{1 / 2} & =\left(R \circ \rho_{\mathrm{Mp}}^{\psi, \Gamma_{1}}(g, q) \circ R^{-1}\right)(h)(0) \\
& =\gamma_{\psi}(q) \cdot \int_{y \in \Gamma}\left(R^{-1} h\right)(y) \mu_{g}^{\psi, \Gamma_{1}}
\end{aligned}
$$

where, for brevity, $\Gamma:=\Gamma_{g^{-1}} / \Gamma_{1} \cap \Gamma_{g^{-1}}$. As in the proof of Lemma A.7, define $P: \bar{V} \oplus V \rightarrow V$ by $P(v, w)=w-v$; it restricts to an isomorphism

$$
P: \Gamma \rightarrow\left(g^{-1}-1\right) V=(g-1) V, \quad P\left(x, g^{-1} x\right):=\left(g^{-1}-1\right) x=(g-1)\left(-g^{-1} x\right) .
$$

We use $P$ to rewrite (29) as an integral over $(g-1) V$.

Let $p: \Gamma \rightarrow \Gamma_{-1}$ be the projection along $\Gamma_{1}$, and $b: V \rightarrow \Gamma_{-1}$ as in (27). Then $P=b^{-1} \circ p$. By (28) and (22) we have, for $y \in \Gamma$,

$$
\begin{aligned}
\left(R^{-1} h\right)(y) & =\left(\operatorname{Res}_{\Gamma_{-1}}^{-1} \circ\left(b^{*}\right)^{-1} h\right)(y)=h(P(y)) \psi\left(\frac{1}{2} \omega(p(y), y-p(y))\right) \\
& =h(P(y)) \psi\left(\frac{1}{2} \omega(p(y), y)\right) .
\end{aligned}
$$

Now (36) gives $\omega(p(y), y)=-Q_{g^{-1}}(P(y), P(y))$. Moreover, it is easy to verify from the definition (35) that $-Q_{g^{-1}}=Q_{g}$. We therefore have

$$
\int_{V} T_{(g, q)}^{\psi} h \mu_{V}^{1 / 2}=\gamma_{\psi}(q) \cdot \int_{v \in(g-1) V} h(v) \psi\left(\frac{1}{2} Q_{g}(v, v)\right) P_{*} \mu_{g}^{\psi, \Gamma_{1}}
$$

and it only remains to argue that $P_{*} \mu_{g}^{\psi, \Gamma_{1}}=\mu_{\sigma_{g}}$.

To do so, note that the natural action of $g$ on (the second factor of) $\bar{V} \oplus V$ fixes $\Gamma_{g^{-1}} \cap \Gamma_{1}$ point-wise. Therefore, following Remark 4.6.1, we conclude that $\mu_{g}^{\psi, \Gamma_{1}}$ is the measure on $\Gamma$ that is self-dual with respect to $\psi \circ q$, where $q$ is the bilinear form $q(x, y)=\omega(x, g y)$. On the other hand, it is elementary to check that $P$ intertwines the forms $q$ and $\sigma_{g}$, that is, $\sigma_{g}(P(x), P(y))=q(x, y)$. Since $\mu_{\sigma_{g}}$ is self-dual for $\psi \circ \sigma_{g}$, we must have $P_{*} \mu_{g, \Gamma_{1}}=\mu_{\sigma_{g}}$ as desired.

\section{Invariant presentation: Proof of Theorem C}

6.1. Now we deduce Theorem $C$. Here is a reformulation of it, in terms of the representation $A_{1}$ of $\mathrm{Mp}(V)$ on $L^{2}(V)$ defined in Section 5.4. (As noted in footnote 6 , we continue to deal with Hilbert spaces of half-densities rather than functions.) 
Proposition 6.2. For any $(g, q) \in \mathrm{Mp}(V)$ and $h \in \mathscr{Y}(V)$,

$$
\begin{aligned}
A_{1}(g, q)(h)(x) & =\int_{v \in V} T_{(g, q)}^{\psi}(v) \psi\left(\frac{1}{2} \omega(v, x)\right) h(x-v) \mu_{V}^{1 / 2} \\
& =:\left(T_{(g, q)}^{\psi} \mu_{V}^{1 / 2} \star h\right)(x) .
\end{aligned}
$$

Proof. Suppose $h$ is Schwartz. Setting $\tilde{h}:=A_{1}(g, q)(h)$, we want to calculate $\tilde{h}(x)$. For any $f \in \mathscr{Y}(V)$ one has $W^{\psi, \ell}(f) \circ \rho_{H}^{\psi, \ell}(x)=W^{\psi, \ell}\left(f_{x}\right)$, where

$$
f_{x}(v):=f(v-x) \psi\left(\frac{1}{2} \omega(v, x)\right) .
$$

According to Proposition 5.2(c),

$$
\tilde{h}(x)=\tilde{h}_{-x}(0)=\operatorname{Tr}\left(W^{\psi, \ell}\left(\tilde{h}_{-x}\right)\right) \cdot \mu_{V}^{1 / 2} .
$$

Unraveling the definitions, we find

$$
\begin{aligned}
\tilde{h}(x) & =\operatorname{Tr}\left(W^{\psi, \ell}(\tilde{h}) \circ \rho_{H}^{\psi, \ell}(-x)\right) \cdot \mu_{V}^{1 / 2} \\
& =\operatorname{Tr}\left(\rho_{\mathrm{Mp}}^{\psi, \ell}(g, q) \circ W^{\psi, \ell}(h) \circ \rho_{H}^{\psi, \ell}(-x)\right) \cdot \mu_{V}^{1 / 2} \\
& =\int_{V} T_{(g, q)}^{\psi} h_{-x} \mu_{V}^{1 / 2}=\int_{v \in V} T_{(g, q)}^{\psi}(v) \psi\left(\frac{1}{2} \omega(v,-x)\right) h(v+x) \mu_{V}^{1 / 2} .
\end{aligned}
$$

Since $T_{(g, q)}^{\psi}$ is an even function on $V$, we obtain the right-hand side of (30).

\section{Transfer factor: Proof of Theorem D}

7.1. First, in Section 7.2, we give a purely algebraic proof, using the central characters to distinguish between $\rho_{+}^{\psi, \ell}$ and $\rho_{-}^{\psi, \ell}$. Then, in Section 7.4, we sketch an alternative argument, because it emphasizes the structure of the Weyl transform, and leads naturally to the geometrization mentioned in Section 1.3. Both methods rely on the following observation.

The decomposition $\rho_{\mathrm{Mp}}^{\psi, \ell}=\rho_{+}^{\psi, \ell} \oplus \rho_{-}^{\psi, \ell}$ into irreducible representations corresponds to the decomposition of the representation space $\mathscr{H}^{\psi, \ell} \cong L^{2}\left(\ell^{\prime}\right)$ into even and odd functions. Let $\Pi: \mathscr{H}^{\psi, \ell} \rightarrow \mathscr{H}^{\psi, \ell}$ be the parity operator defined by

$$
(\Pi f)(x)=f(-x) \text {. }
$$

Then, as generalized functions on $\operatorname{Mp}(V)$,

$$
\operatorname{Tr} \rho_{ \pm}^{\psi, \ell}(g, q)=\frac{1}{2} \operatorname{Tr}\left(\rho_{\mathrm{Mp}}^{\psi, \ell}(g, q) \pm \rho_{\mathrm{Mp}}^{\psi, \ell}(g, q) \circ \Pi\right)
$$

whence

$$
\operatorname{Tr}\left(\rho_{+}^{\psi, \ell}-\rho_{-}^{\psi, \ell}\right)(g, q)=\operatorname{Tr}\left(\rho_{\mathrm{Mp}}^{\psi, \ell}(g, q) \circ \Pi\right) .
$$


7.2. “Algebraic" proof. The representations $\rho_{+}^{\psi, \ell}$ and $\rho_{-}^{\psi, \ell}$ have different central characters, and this can be used to distinguish them. Concretely, the central element $(-1,1) \in \operatorname{Mp}^{\psi, \ell}(V)$ acts as $\Pi$ on $\mathscr{H}^{\psi, \ell}$. Given $(g, \xi) \in \operatorname{Mp}^{\psi, \ell}(V)$, one has $(g, \xi)(-1,1)=(-g, \xi) \in \operatorname{Mp}^{\psi, \ell}(V)$, and therefore

$$
\left(\operatorname{Tr} \rho_{+}^{\psi, \ell}-\operatorname{Tr} \rho_{-}^{\psi, \ell}\right)(g, \xi)=\operatorname{Tr} \rho_{\mathrm{Mp}}^{\psi, \ell}(-g, \xi) .
$$

On the other hand, if in the notation of Section 3.3 we have $\alpha_{\ell}(g, q)=(g$, $\xi)$, then $\alpha_{\ell}^{-1}(-g, \xi)=\left(-g, q+\epsilon_{g}\right)$ as elements of $\operatorname{Mp}(V)$, where

$$
\epsilon_{g}:=\tau\left(\ell \oplus \ell, \Gamma_{1}, \Gamma_{g}, \ell \oplus g \ell\right)-\tau\left(\ell \oplus \ell, \Gamma_{1}, \Gamma_{-g}, \ell \oplus(-g) \ell\right) .
$$

Since the central factor $W(F) / I^{3} \subset \operatorname{Mp}(V)$ acts through $\gamma_{\psi}$, we have

$$
\left(\operatorname{Tr} \rho_{+}^{\psi, \ell}-\operatorname{Tr} \rho_{-}^{\psi, \ell}\right)(g, q)=\operatorname{Tr} \rho\left(-g, q+\epsilon_{g}\right)=\operatorname{Tr} \rho^{\psi, \ell}(-g, q) \cdot \gamma_{\psi}\left(\epsilon_{g}\right) .
$$

Thus it remains to prove the following lemma, which relies on the combinatorics of the Maslov index.

Lemma 7.3. One has $\epsilon_{g}=Q_{g}$ in $W(F)$.

Proof. Consider the polyhedron with two triangular and two quadrilateral faces:

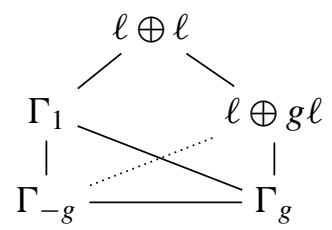

As explained in Section A.5.2, the sum of the Maslov indices of the faces vanishes. The sum over the two quadrilateral faces is $\epsilon_{g}$ (note that $(-g) \ell=g \ell$ ); therefore

$$
\epsilon_{g}=\tau\left(\Gamma_{-g}, \Gamma_{1}, \Gamma_{g}\right)+\tau\left(\Gamma_{g}, \ell \oplus g \ell, \Gamma_{-g}\right) .
$$

The second term must vanish, since

$$
\tau\left(\Gamma_{g}, \ell \oplus g \ell, \Gamma_{-g}\right)=-\tau\left(\Gamma_{-g}, \ell \oplus g \ell, \Gamma_{g}\right)=-\tau\left(\Gamma_{g}, \ell \oplus g \ell, \Gamma_{-g}\right)
$$

by Section A.5(a) and (d) applied to

$$
1 \oplus(-1) \in \mathrm{GL}(\bar{V} \oplus V) .
$$

The first term $\tau\left(\Gamma_{-g}, \Gamma_{1}, \Gamma_{g}\right)$ equals $\tau\left(\Gamma_{1}, \Gamma_{g}, \Gamma_{-1}\right)$ by Section A.5(d) applied to $(x, y) \mapsto\left(g^{-1} y, x\right)$, with $\lambda=-1$; but Lemma A.7 says that $\tau\left(\Gamma_{1}, \Gamma_{g}, \Gamma_{-1}\right)$ is the class of $Q_{g}$. 


\section{4. “Analytic” proof.}

Lemma 7.5. For any $h \in \mathscr{S}(V)$, we have $W^{\psi, \ell}(h) \circ \Pi=W^{\psi, \ell}(\mathfrak{F} h)$, where

$$
\mathfrak{F}: L^{2}(V) \rightarrow L^{2}(V)
$$

is the Fourier transform

$$
(\mathfrak{F} h)(x):=\|2\|^{-\frac{\operatorname{dim} V}{2}} \int_{v \in V} h(v) \psi\left(\frac{1}{2} \omega(v, x)\right) \mu_{V} .
$$

Moreover, $\Pi \circ W^{\psi, \ell}(h) \circ \Pi=W^{\psi, \ell}(\Pi h)$ where $\Pi h(v):=h(-v)$.

Proof. The last statement follows directly from (26). From there, too, one sees that $W^{\psi, \ell}(h) \circ \Pi$ is represented by the kernel $A^{*} \circ B^{*} \circ\left(\mathfrak{F}_{0} \otimes \mathrm{id}\right)(h)$, where $B(a, b)=(b, a)$. The result then follows from the commutativity of the diagram

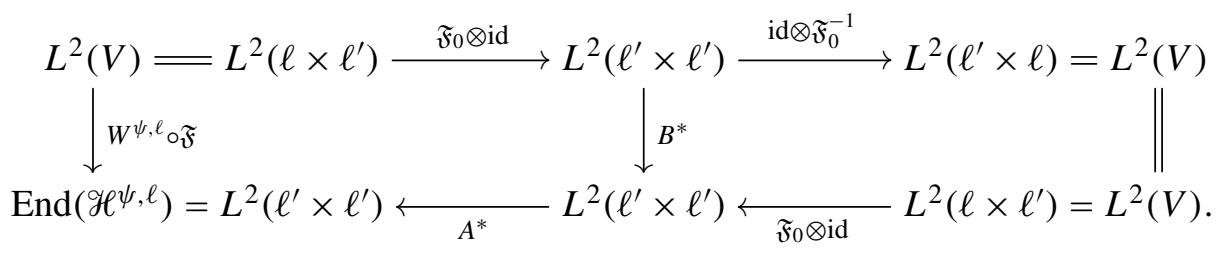

Here the top row composes to $\mathfrak{F}$ and the bottom row to $W^{\psi, \ell}$ by Lemma 5.3.

Now to deduce Theorem D. For brevity, we detail only the case when $F$ is finite, but the infinite case is parallel. Applying the formula for $\operatorname{Tr} \rho_{\mathrm{Mp}}^{\psi, \ell}$ from Corollary 1.4, the claim is that

$$
\operatorname{Tr}\left(\rho_{+}^{\psi, \ell}-\rho_{-}^{\psi, \ell}\right)(g, q)=\sqrt{\# V^{-g}} \cdot \gamma_{\psi}(q) \gamma_{\psi}\left(Q_{g}\right) .
$$

By Theorem C, $\rho_{\mathrm{Mp}}^{\psi, \ell}(g, q)$ is the Weyl transform $W^{\psi, \ell}\left(T_{(g, q)}^{\psi} \mu_{V}^{1 / 2}\right)$, so (31), Lemma 7.5, and Proposition 5.2(c) give

$$
\begin{aligned}
\operatorname{Tr}\left(\rho_{+}^{\psi, \ell}-\rho_{-}^{\psi, \ell}\right)(g, q) & =\operatorname{Tr}\left(\rho_{\mathrm{Mp}}^{\psi, \ell}(g, q) \circ \Pi\right) \\
& =\operatorname{Tr} W^{\psi, \ell}\left(\mathfrak{F}\left(T_{(g, q)}^{\psi} \mu_{V}^{1 / 2}\right)\right)=\mathfrak{F}\left(T_{(g, q)}^{\psi}\right)(0) .
\end{aligned}
$$

The result now follows from Theorem B and the definition of $\gamma_{\psi}$ in Section A.3. In detail:

$$
\begin{aligned}
\mathfrak{F}\left(T_{(g, q)}^{\psi}\right)(0) & =\gamma_{\psi}(q) \cdot \int_{v \in V} \psi\left(\frac{1}{2} Q_{g}(v, v)\right) \cdot D_{g}^{\psi} \cdot \mu_{V} & & \text { (by Thm B) } \\
& =\gamma_{\psi}(q) \cdot \int_{v \in(g-1) V} \psi\left(\frac{1}{2} Q_{g}(v, v)\right) \cdot \mu_{\sigma_{g}} & & \text { (by def. of } \left.D_{g}^{\psi}\right) \\
& =\gamma_{\psi}(q) \cdot M \int_{v \in(g-1) V / V^{-g}} \psi\left(\frac{1}{2} Q_{g}(v, v)\right) \cdot \mu_{Q_{g}} & & \text { (see below) } \\
& =M \gamma_{\psi}(q) \gamma_{\psi}\left(Q_{g}\right) & & \text { (by def. of } \left.\gamma_{\psi}\right) .
\end{aligned}
$$


To explain the third line, there is a unique measure $\mu$ on $V^{-g}$ such that $\mu_{\sigma_{g}}$ is a product measure $\mu_{\sigma_{g}}=\mu \otimes \mu_{Q_{g}}$, and then $M:=\int_{V^{-g}} \mu$. However, a self-dual measure on a vector space $X$ is always $1 / \sqrt{\# X}$ times counting measure; this implies that $M=\sqrt{\# V^{-g}}$, and the proof of (32) is complete.

\section{Appendix: Witt, Weil, Maslov, Cayley}

A.1. Witt group. (The basic reference is [Lam 2005].) Let $F$ be a field of characteristic not 2. A quadratic space is a pair $(W, q)$, where $W$ is a finite-dimensional vector space over $F$ and $q: W \otimes W \rightarrow F$ is a nondegenerate symmetric bilinear form. The perpendicular direct sum and the tensor product of two quadratic spaces can be defined in an obvious way. With these operations, the set of isomorphism classes of quadratic spaces forms a commutative semiring. The Witt group (or ring) $W(F)$ is the commutative ring defined by imposing the relation

$$
(W, q)+(W,-q)=0 .
$$

The dimension (or rank) of a quadratic space $(W, q)$ is $\operatorname{dim} W \in \mathbb{Z}$. The discriminant of $(W, q)$ is defined as follows. First, $q$ defines a symmetric map $\Phi: W \rightarrow W^{*}$ such that $q(x, y)=\Phi(x)(y)$. Suppose $e_{1}, \ldots, e_{n}$ is a basis for $W$, and $e_{1}^{*}, \ldots, e_{n}^{*}$ the dual basis for $W^{*}: e_{i}^{*}\left(e_{j}\right)=\delta_{i j}$. Then $\operatorname{det} q \in F$ is the scalar such that

$$
\Phi e_{1} \wedge \cdots \wedge \Phi e_{n}=(\operatorname{det} q)\left(e_{1}^{*} \wedge \cdots \wedge e_{n}^{*}\right) \in \wedge^{n} W^{*} .
$$

The class of $\operatorname{det} q$ in $F^{\times} /\left(F^{\times}\right)^{2}$ is well defined, and is called the disciminant of $(W, q)$. The signed discriminant $\operatorname{sdet} q$ of $(W, q)$ is $(-1)^{n(n-1) / 2} \operatorname{det} q \in F^{\times} /\left(F^{\times}\right)^{2}$.

Define a commutative ring $W_{0}(F)$ to be $\mathbb{Z} / 2 \mathbb{Z} \times F^{\times} /\left(F^{\times}\right)^{2}$ as a set, with the operations

$$
\begin{aligned}
\left(d_{1}, \Delta_{1}\right)+\left(d_{2}, \Delta_{2}\right) & :=\left(d_{1}+d_{2},(-1)^{d_{1} d_{2}} \Delta_{1} \Delta_{2}\right), \\
\left(d_{1}, \Delta_{1}\right)\left(d_{2}, \Delta_{2}\right) & :=\left(d_{1} d_{2}, \Delta_{1}^{d_{2}} \Delta_{2}^{d_{1}}\right) .
\end{aligned}
$$

The dimension and signed discriminant together define a surjective homomorphism

$$
\widetilde{Q}=(\operatorname{dim}, \text { sdet }): W(F) \rightarrow W_{0}(F) .
$$

Let $I \subset W(F)$ be the kernel $I=\operatorname{ker}(\operatorname{dim})$. Then $\operatorname{ker} \widetilde{Q}=I^{2}$; see $[$ Lam 2005, Chapter 2, Proposition 2.1]. In other words, $\widetilde{Q}$ identifies $W(F) / I^{2}$ with $W_{0}(F)$.

Remark A.1.1. The dimension and signed discriminant make sense for any nondegenerate bilinear form, symmetric or not. Such a form $q$ therefore defines a class $[q]$ in $W_{0}(F)=W(F) / I^{2}$. 
A.1.2. Finite and local fields. We want to describe $W(F) / I^{3}$, in case $F$ is a finite or local field. For $a, b \in F^{\times}$, the Hilbert symbol $(a, b)_{H}$ is defined to equal 1 if $a$ is a norm from $F(\sqrt{b})$, and to equal -1 if not. Let $Z_{F}$ be the image of the Hilbert symbol; it is either $Z_{F}=\{ \pm 1\}$ (when $F$ is real or nonarchimedean) or $Z_{F}=\{1\}$ (when $F$ is finite or complex). The Hasse invariant $s(q) \in\{ \pm 1\}$ of a quadratic space $(W, q)$ over $F$ can be defined inductively by $s\left(q \oplus q^{\prime}\right)=s(q) s\left(q^{\prime}\right)\left(\operatorname{det} q, \operatorname{det} q^{\prime}\right)_{H}$, and $s(q)=1$ if $\operatorname{dim} q=1$.

Theorem A.2. Let $F$ be any finite or local field of characteristic not 2. Two classes in $W(F)$ are equal modulo $I$ if and only if they can be represented by quadratic spaces of the same rank. Two quadratic spaces of the same rank have the same class modulo $I^{2}$ if and only if they have the same discriminant. Two quadratic spaces of the same rank and discriminant have the same class modulo $I^{3}$ if and only if they have the same Hasse invariant; moreover, $I^{2} / I^{3}$ is canonically isomorphic to $Z_{F}$.

Proof. For the first statement, every class in $W(F)$ is represented by some quadratic space; see, e.g., [Lam 2005, Chapter 2, Proposition 1.4(1)]. If our two classes are represented by $(W, q)$ and $\left(W^{\prime}, q^{\prime}\right)$, with $\operatorname{dim} W-\operatorname{dim} W^{\prime}=2 m \geq 0$, let $\left(W_{0}, q_{0}\right)$ be any quadratic space of rank $m$. Then $q^{\prime} \oplus q_{0} \oplus\left(-q_{0}\right)$ has the same class as $q^{\prime}$ and the same rank as $q$. The second statement follows from the isomorphism $\widetilde{Q}: W(F) / I^{2} \rightarrow W_{0}(F)$. (The argument so far does not use the assumption that $F$ is finite or local.)

For the third statement, we use the fact that two quadratic spaces of the same dimension have the same class in $W(F)$ if and only if they are isometric [Lam 2005, Chapter 2, Proposition 1.4(3)]. There are four cases.

First, suppose $F$ is nonarchimedean local. Then two quadratic spaces are isometric if and only if they have the same rank, discriminant, and Hasse invariant [Lam 2005, Chapter 6, Theorem 2.12]; moreover, $I^{3}=0$ [Lam 2005, Chapter 6, Corollary 2.15]. So two quadratic spaces of the same rank have the same class in $W(F)=W(F) / I^{3}$ if and only if they have the same discriminant and Hasse invariant.

Second, suppose $F=\mathbb{F}_{q}$. This time quadratic spaces are isometric if and only if they have the same rank and discriminant [Lam 2005, Chapter 2, Thdorem 3.5]; the Hasse invariant (like the Hilbert symbol) always equals 1. From this it follows that $I^{3}=I^{2}=0$, and we can argue as for the nonarchimedean local case.

Third, suppose $F=\mathbb{C}$. Now two quadratic spaces are isometric if and only if they have the same rank; the discriminant and Hasse invariant (like the Hilbert symbol) always equal 1 . This time $I^{3}=I=0$, and we can argue as before.

Fourth, suppose $F=\mathbb{R}$. Isomorphism classes of quadratic spaces are classified by pairs $\left(n_{+}, n_{-}\right)$of nonnegative integers, $n_{ \pm}$being the dimension of the largest positive/negative-definite subspace. The "signature" sig: $\left(n_{+}, n_{-}\right) \mapsto n_{+}-n_{-}$ 
defines an isomorphism $W(F) \rightarrow \mathbb{Z}$, identifying $I$ with $2 \mathbb{Z}$ and $I^{3}$ with $8 \mathbb{Z}$. (For all this see [Lam 2005, Chapter 2, Proposition 3.2]. One finds that the rank is $\operatorname{dim}\left(n_{+}, n_{-}\right)=n_{+}+n_{-}, \operatorname{det}\left(n_{+}, n_{-}\right)=(-1)^{n_{-}}$, and $s\left(n_{+}, n_{-}\right)=(-1)^{n_{-}\left(n_{-}-1\right) / 2}$. It follows that the rank and signed discriminant determine the signature mod 4 , and that for fixed rank and discriminant, the two choices of Hasse invariant correspond to the two choices of signature mod 8.

For the last statement, it is formally only necessary to show that $I^{2} / I^{3}$ and $Z_{F}$ have the same number of elements, which follows from the above considerations; however, we will explain the isomorphism using the Weil index - see A.4.1(d) below.

A.3. Weil index. In this section, let $F$ be a finite or local field of characteristic not 2. The Weil index is a homomorphism $\gamma_{\psi}: W(F) \rightarrow Z_{8}$, where $Z_{8} \subset \mathbb{C}^{\times}$is the group of eighth roots of unity. It is defined using Fourier transforms.

A.3.1. Densities and measures. First let us recall some facts about measures and densities that will be useful both here and in the main text. A nice introduction to densities can be found in [Woodhouse 1980, §5.9].

For $s \in \mathbb{R}$, and $X$ any finite-dimensional vector space over $F$, let $\Omega_{s}(X)$ denote the space of complex translation-invariant $s$-densities on $X$; it is a one-dimensional complex vector space, the complexification of the space of real translation-invariant $s$-densities. In particular, there is a canonical isomorphism

$$
\Omega_{1 / 2}(X) \otimes_{\mathbb{C}} \Omega_{1 / 2}(X) \rightarrow \Omega_{1}(X),
$$

and every positive invariant density (that is, Haar measure) $\mu \in \Omega_{1}(X)$ has a canonical square root $\mu^{1 / 2} \in \Omega_{1 / 2}(X)$. The space of functions $X \rightarrow \Omega_{1 / 2}(X)$ has a natural Hermitian inner product:

$$
\left(f_{1}, f_{2}\right):=\int_{X} \overline{f_{1}} f_{2}
$$

considering $\overline{f_{1}} f_{2}: X \rightarrow \Omega_{1 / 2}(X) \otimes \Omega_{1 / 2}(X)=\Omega_{1}(X)$ as a density on $X$. Let $L^{2}(X)$ denote the corresponding Hilbert space.

A perfect pairing $B: X \otimes_{F} Y \rightarrow U(1)$ (making $X$ the Pontryagin dual of $Y$ ) associates to each nonzero $\mu \in \Omega_{1}(X)$ a dual measure $\mu^{*} \in \Omega_{1}(Y)$. It can be usefully characterized by the Fourier inversion formula $\left(\mathfrak{F}_{B^{*}}^{\mu^{*}} \mathfrak{F}_{B}^{\mu} f\right)(z)=f(-z)$ for all Schwartz functions $f: X \rightarrow \mathbb{C}$. Here

$$
\left(\mathfrak{F}_{B}^{\mu} f\right)(y)=\int_{x \in X} f(x) B(x, y) \mu
$$

and $B^{*}(y, x):=B(x, y)$ for all $(x, y) \in X \times Y$.

If $Y=X$ then there is a unique self-dual $\mu \in \Omega_{1}(X)$ such that $\mu^{*}=\mu$. Of particular interest is the situation where $B=B_{q}^{\psi}:=\psi \circ q$ for some nontrivial, 
continuous homomorphism $\psi: F \rightarrow U(1)$ and some nondegenerate bilinear form $q: X \otimes_{F} X \rightarrow F$. It is easy to see from the Fourier inversion formula that if $\mu_{q}^{\psi}$ is self-dual for $B_{q}^{\psi}$, then the measure that is self-dual for $B_{a q}^{\psi}, a \in F^{\times}$, is $\mu_{a q}^{\psi}=\|a\|^{(\operatorname{dim} X) / 2} \mu_{q}^{\psi}$.

A.3.2. Definition. Suppose now that $(X, q)$ is a quadratic space (that is, $q$ is from now on symmetric). We fix a nontrivial, continuous homomorphism $\psi: F \rightarrow U(1)$ and write $f_{q}^{\psi}$ for the function $f_{q}^{\psi}(x)=\psi\left(\frac{1}{2} q(x, x)\right)$.

Theorem A.4 [Weil 1964, Theorem 2 and Proposition 3]. There exists a number $\gamma_{\psi}(q) \in Z_{8}$ such that

$$
\mathfrak{F}_{B_{q}^{\psi}}^{\mu_{q}^{\psi}} f_{q}^{\psi}=\gamma_{\psi}(q) \cdot f_{-q}^{\psi}
$$

as generalized functions on $X$. Moreover, $(X, q) \mapsto \gamma_{\psi}(q)$ defines a character $\gamma_{\psi} \psi: W(F) \rightarrow Z_{8}$

Note that $f_{q}^{\psi}$ is not Schwartz, but its Fourier transform can be defined in the sense of distributions.

A.4.1. Properties. The following properties of $\gamma_{\psi}$ are used in this paper, and go back to [Weil 1964]. For $a \in F^{\times}$, let $q_{a}$ be the bilinear form $q_{a}(x, y)=a x y$ on $F$, and write $\gamma_{\psi}(a):=\gamma_{\psi}\left(q_{a}\right)$. We again write $(\cdot, \cdot)_{H}$ for the Hilbert symbol, $Z_{F}$ for its image, and $s(q) \in Z_{F}$ for the Hasse invariant of any quadratic space $(W, q)$ (see Section A.1.2).

(a) If $\psi^{\prime}(x)=\psi(a x)$, then $\gamma_{\psi}\left(q_{a} \otimes q\right)=\gamma_{\psi^{\prime}}(q)$.

(b) $\gamma_{\psi}(a) \gamma_{\psi}(b)=\gamma_{\psi}(1) \gamma_{\psi}(a b)(a, b)_{H}$.

(c) $\gamma_{\psi}(q)=\gamma_{\psi}(1)^{\operatorname{dim} q-1} \gamma_{\psi}(\operatorname{det} q) s(q)$.

(d) $\gamma_{\psi}$ is trivial on $I^{3} \subset W(F)$, and $\gamma_{\psi}$ restricts to an isomorphism $I^{2} / I^{3} \rightarrow Z_{F}$.

Proofs. Statement (a) follows easily from the definition of $\gamma_{\psi}$ in Theorem A.4 (note that $f_{q_{a} \otimes q}^{\psi}=f_{q}^{\psi^{\prime}}, B_{q_{a} \otimes q}^{\psi}=B_{q}^{\psi^{\prime}}, \mu_{q_{a} \otimes q}^{\psi}=\mu_{q}^{\psi^{\prime}}$ ). Statement (b) is equivalent to the last formula on p. 176 of [Weil 1964]. Statement (c) follows from (b) by induction on the dimension (that is, if we decompose $q$ as a perpendicular sum of two smaller spaces). The first part of statement (d) follows from Theorem A.2: if two classes in $W(F)$ are equal modulo $I^{3}$, then they can be represented by spaces of the same rank, discriminant, and Hasse invariant, and so by (c) have the same Weil index. For the second part of (d), set $q_{a, b}=\left(q_{1} \oplus q_{-a}\right) \otimes\left(q_{1} \oplus q_{-b}\right)=q_{1} \oplus q_{-a} \oplus q_{-b} \oplus q_{a b}$, for any $a, b \in F^{\times} ; I^{2}$ is generated by forms of this type [Lam 2005, Chapter 2, Proposition 1.2]. By (b), $\gamma_{\psi}\left(q_{a, b}\right)=(a, b)_{H}$, so indeed $\gamma_{\psi}\left(I^{2}\right)=Z_{F}$. To see that $I^{3}$ is the kernel of $\gamma_{\psi}$ on $I^{2}$, recall from Theorem A.2 that any two classes in $I^{2}$ can be represented by quadratic spaces $(W, q),\left(W^{\prime}, q^{\prime}\right)$ of the same rank and 
discriminant; according to (c), $\gamma_{\psi}(q)=\gamma_{\psi}\left(q^{\prime}\right)$ if and only if $s(q)=s\left(q^{\prime}\right)$, in other words (again according to Theorem A.2) if and only if $q=q^{\prime} \bmod I^{3}$.

A.5. Maslov index. In this section, let $F$ be any field of characteristic not 2. Let $(V, \omega)$ be a finite-dimensional symplectic vector space over $F$. The Maslov index $\tau$ associates to each arbitrary sequence $\ell_{1}, \ldots, \ell_{n} \subset V$ of Lagrangian subspaces, a class $\tau\left(\ell_{1}, \ldots, \ell_{n}\right)$ in $W(F)$. It is characterized by the following properties:

(a) Dihedral symmetry:

$$
\tau\left(\ell_{1}, \ldots, \ell_{n}\right)=-\tau\left(\ell_{n}, \ldots, \ell_{1}\right)=\tau\left(\ell_{n}, \ell_{1}, \ldots, \ell_{n-1}\right) .
$$

(b) Chain condition: For any $j, 1<j<n$,

$$
\tau\left(\ell_{1}, \ell_{2}, \ldots, \ell_{j}\right)+\tau\left(\ell_{1}, \ell_{j}, \ldots, \ell_{n}\right)=\tau\left(\ell_{1}, \ell_{2}, \ldots, \ell_{n}\right) .
$$

(c) Additivity: If $V, V^{\prime}$ are symplectic spaces, $\ell_{1}, \ldots, \ell_{n} \in \operatorname{Lagr}(V), \ell_{1}^{\prime}, \ldots, \ell_{n}^{\prime} \in$ $\operatorname{Lagr}\left(V^{\prime}\right)$, so that $\ell_{i} \oplus \ell_{i}^{\prime} \in \operatorname{Lagr}\left(V \oplus V^{\prime}\right)$, then we have

$$
\tau\left(\ell_{1} \oplus \ell_{1}^{\prime}, \ldots, \ell_{n} \oplus \ell_{n}^{\prime}\right)=\tau\left(\ell_{1}, \ldots, \ell_{n}\right)+\tau\left(\ell_{1}^{\prime}, \ldots, \ell_{n}^{\prime}\right) .
$$

(d) Invariance: Suppose $g \in \operatorname{GL}(V)$ satisfies $\omega(g x, g y)=\lambda \omega(x, y)$ for all $x, y \in V$. Then

$$
\tau\left(g \ell_{1}, \ldots, g \ell_{n}\right)=q_{\lambda} \otimes \tau\left(\ell_{1}, \ldots, \ell_{n}\right)
$$

where $q_{\lambda} \in W(F)$ is the bilinear form on $F$ defined by $(x, y) \mapsto \lambda x y$.

(e) $\tau\left(\ell_{1}, \ell_{2}, \ell_{3}\right)$ can be represented by the (possibly degenerate) bilinear form on $\ell_{2} \cap\left(\ell_{1}+\ell_{3}\right)$ given by $(x, y) \mapsto \omega\left(x, y_{3}\right)$ (where $y=y_{1}+y_{3}$ with $\left.y_{i} \in \ell_{i}\right)$.

For a definition and proofs of (a) and (b), see [Thomas 2006]; (c), (d), and (e) are simple consequences of the definition given there.

A.5.1. Rank and discriminant. The rank and discriminant were calculated in [Parimala et al. 2000, Proposition 2.1], with the following result. For each Lagrangian $\ell$, choose an "orientation" $o$, that is, a nonzero element of $\operatorname{det}(\ell)$, the top exterior power of $\ell$. Given $(\ell, o),\left(\ell^{\prime}, o^{\prime}\right)$, choose an isomorphism $\alpha: \ell \rightarrow \ell^{\prime}$ such that $\alpha$ is the identity on $\ell \cap \ell^{\prime}$, and $\alpha_{*}(o)=o^{\prime}$. Consider the nondegenerate bilinear form $q(x, y)=\omega(\alpha(x), y)$ on $\ell / \ell \cap \ell^{\prime}$. Set

$$
Q\left(\ell, o ; \ell^{\prime}, o^{\prime}\right)=[q] \in W(F) / I^{2}
$$

(in the notation of Remark A.1.1). It is easy to check that $Q\left(\ell, o ; \ell^{\prime}, o^{\prime}\right)$, unlike $q$, is independent of the choice of $\alpha$; moreover, $Q\left(\ell^{\prime}, o^{\prime} ; \ell, o\right)=-Q\left(\ell, o ; \ell^{\prime}, o^{\prime}\right)$. What [Parimala et al. 2000] show is that, for any choice of orientations $o_{i} \in \operatorname{det} \ell_{i}$,

$$
\tau\left(\ell_{1}, \ldots, l_{n}\right)=\sum_{i \in \mathbb{Z} / n \mathbb{Z}} Q\left(\ell_{i}, o_{i} ; \ell_{i+1}, o_{i+1}\right) \bmod I^{2} .
$$


A.5.2. Polygons and polyhedra. Properties (a) and (b) deserve further comment. Suppose given an oriented $n$-sided polygon $F$ with vertices $\ell_{1}, \ldots, \ell_{n}$. The dihedral symmetry (a) allows us to unambiguously define $\tau(F)=\tau\left(\ell_{1}, \ldots, \ell_{n}\right)$; reversing the orientation of the polygon reverses the sign of $\tau(F)$. The chain condition (b) has the following interpretation: suppose that $P$ is a closed, oriented polyhedron with vertices $\ell_{1}, \ldots, \ell_{n}$. Then (b) implies that

$$
\sum_{F} \tau(F)=0
$$

where the sum is over the faces $F$ of $P$.

A.6. Cayley transform. We continue with any field $F$ of characteristic not 2. Let $(V, \omega)$ be a finite-dimensional symplectic vector space over $F$.

A.6.1. Formulas. For all $g \in \operatorname{Sp}(V)$ there is a symmetric form $Q$ on $V$ given by

$$
Q(x, y)=\frac{1}{2} \omega((g+1) x,(g-1) y) .
$$

The kernel is $V^{g}+V^{-g}$ (a direct sum in $V$ ). The corresponding map

$$
\operatorname{Sp}(V) \rightarrow \operatorname{Sym}^{2}\left(V^{*}\right)=\mathfrak{s p}(V)
$$

is the Cayley transform (usually defined without the factor $\frac{1}{2}$ ); it is traditionally formulated [Cayley 1846] as a bijection between the open subsets of $\operatorname{Sp}(V)$ and $\mathfrak{s p}(V)$ defined (in both cases) by the condition $\operatorname{det}(g-1) \neq 0$.

The canonical isomorphism $V / V^{g} \rightarrow(g-1) V$ transfers $Q$ to a symmetric form $Q_{g}$ on $(g-1) V$, with kernel $V^{-g}$. This is the form used in the main text:

$$
Q_{g}((g-1) x,(g-1) y):=\frac{1}{2} \omega((g+1) x,(g-1) y) \text { for all } x, y \in V .
$$

It is easy to check that $Q_{g}=-Q_{g^{-1}}=Q_{-g^{-1}}$.

A.6.2. The Cayley form as a Maslov index. Let $\bar{V}$ be the same vector space $V$, but equipped with symplectic form $-\omega$. For $g \in \operatorname{Sp}(V)$, we write $\Gamma_{g}$ for the graph $\Gamma_{g}=\{(v, g v) \mid v \in V\}$ considered as a Lagrangian subspace of the symplectic vector space $\bar{V} \oplus V$.

Lemma A.7. The class of $Q_{g}$ in the Witt group $W(F)$ equals the Maslov in$\operatorname{dex} \tau\left(\Gamma_{1}, \Gamma_{g}, \Gamma_{-1}\right)$.

Proof. Let $p: \bar{V} \oplus V \rightarrow \Gamma_{-1}$ be the projection along $\Gamma_{1}$. According to Section A.5(e), $\tau\left(\Gamma_{1}, \Gamma_{g}, \Gamma_{-1}\right)$ can be represented by the degenerate symmetric bilinear form on $\Gamma_{g}$ defined by

$$
q(x, y)=\omega(x, p(y)) .
$$


Now consider the map $P: \bar{V} \oplus V \rightarrow V$ given by $P(v, w)=w-v$. We have the following more precise claim, which is easy to check: $P$ induces an isomorphism $\Gamma_{g} / \Gamma_{1} \cap \Gamma_{g} \rightarrow(g-1) V$ that is an isometry between $q$ and $Q_{g}$. In particular,

$$
Q_{g}(P(x), P(y))=\omega(x, p(y)) .
$$

This concludes the proof.

\section{Acknowledgments}

I am grateful to Jeff Adams for discussing his work with me and posing some interesting questions, to Vladimir Drinfeld for emphasizing to me the importance of the Weyl transform, to Maurice de Gosson and Amritanshu Prasad for sharing their results with me, and to Masoud Kamgarpour for many useful discussions. While preparing this paper I was supported by a Seggie Brown Fellowship from the University of Edinburgh.

\section{References}

[Adams 1998] J. Adams, "Lifting of characters on orthogonal and metaplectic groups", Duke Math. J. 92:1 (1998), 129-178. MR 99h:22014 Zbl 0983.11025

[Cayley 1846] A. Cayley, "Sur quelques propriétés des déterminants gauches", J. Reine Angew. Math. 32 (1846), 119-123.

[de Gosson and Luef 2009] M. de Gosson and F. Luef, "The pseudo-character of the Weil representation and its relation with the Conley-Zehnder Index”, preprint, 2009. arXiv 0909.1437

[Grove 2001] L. C. Grove, Classical groups and geometric algebra, Graduate Studies in Mathematics 39, American Mathematical Society, Providence, RI, 2001. MR 2002m:20071 Zbl 0990.20001

[Gurevich and Hadani 2007] S. Gurevich and R. Hadani, "The geometric Weil representation", Selecta Math. (N.S.) 13:3 (2007), 465-481. MR 2009e:11078 Zbl 1163.22004

[Harish-Chandra 1954] Harish-Chandra, "Representations of semisimple Lie groups. III", Trans. Amer. Math. Soc. 76 (1954), 234-253. MR 16,11e Zbl 0055.34002

[Howe 1973] R. Howe, "Invariant theory and duality for classical groups over finite fields", preprint, Yale University, 1973.

[Hua 1948] L.-K. Hua, "On the automorphisms of the symplectic group over any field", Ann. of Math. (2) 49 (1948), 739-759. MR 10,352e

[Lafforgue and Lysenko 2009] V. Lafforgue and S. Lysenko, "Geometric Weil representation: local field case”, Compos. Math. 145:1 (2009), 56-88. MR 2010c:22024 Zbl 1220.22015

[Lam 2005] T. Y. Lam, Introduction to quadratic forms over fields, Graduate Studies in Mathematics 67, American Mathematical Society, Providence, RI, 2005. MR 2005h:11075 Zbl 1068.11023

[Lion and Vergne 1980] G. Lion and M. Vergne, The Weil representation, Maslov index and theta series, Progress in Mathematics 6, Birkhäuser, Mass., 1980. MR 81j:58075 Zbl 0444.22005

[Maktouf 1999] K. Maktouf, "Le caractère de la représentation métaplectique et la formule du caractère pour certaines représentations d'un groupe de Lie presque algébrique sur un corps $p$ adique", J. Funct. Anal. 164:2 (1999), 249-339. MR 2000f:22022 Zbl 0948.22017 
[Parimala et al. 2000] R. Parimala, R. Preeti, and R. Sridharan, "Maslov index and a central extension of the symplectic group”, K-Theory 19:1 (2000), 29-45. MR 2001c:11053a Zbl 1037.11026

[Perrin 1981] P. Perrin, "Représentations de Schrödinger, indice de Maslov et groupe metaplectique", pp. 370-407 in Noncommutative harmonic analysis and Lie groups (Marseille, 1980), edited by J. Carmona and M. Vergne, Lecture Notes in Math. 880, Springer, Berlin, 1981. MR 83m:22027 Zbl 0462.22008

[Prasad 2009] A. Prasad, "On character values and decomposition of the Weil representation associated to a finite abelian group", J. Anal. 17 (2009), 73-85. MR 2012a:11053 Zbl 05924869

[Prasad 2011] A. Prasad, "An easy proof of the Stone-von Neumann-Mackey theorem", Expo. Math. 29:1 (2011), 110-118. MR 2012f:22003 Zbl 1227.43009

[Sliman 1984] M. H. Sliman, Théorie de Mackey pour les groupes adéliques, Astérisque 115, Société Mathématique de France, Paris, 1984. MR 86b:22033 Zbl 0552.22001

[Suslin 1987] A. A. Suslin, “Torsion in $K_{2}$ of fields”, K-Theory 1:1 (1987), 5-29. MR 89a:11123 Zbl 0635.12015

[Thomas 2006] T. Thomas, "The Maslov index as a quadratic space", Math. Res. Lett. 13:5-6 (2006), 985-999. MR 2007j:53093 arXiv 0505561 (expanded version)

[Thomas 2008] T. Thomas, "The character of the Weil representation", J. Lond. Math. Soc. (2) 77:1 (2008), 221-239. MR 2008k:11049 Zbl 1195.11058

[Weil 1964] A. Weil, “Sur certains groupes d'opérateurs unitaires”, Acta Math. 111 (1964), 143-211. MR 29 \#2324 Zbl 0203.03305

[Woodhouse 1980] N. Woodhouse, Geometric quantization, Oxford Mathematical Monographs, The Clarendon Press, New York, 1980. MR 84j:58058 Zbl 0458.58003

Communicated by Marie-France Vignéras

Received 2011-08-14 Revised 2012-06-05 Accepted 2012-07-05

jtthomas@uchicago.edu School of Mathematics, University of Edinburgh, JCMB, The King's Buildings, Mayfield Road, Edinburgh, EH9 3JZ, United Kingdom 


\section{Algebra \& Number Theory}

msp.org/ant

\section{EDITORS}

MANAGING EDITOR

Bjorn Poonen

Massachusetts Institute of Technology

Cambridge, USA

\author{
EDITORIAL BOARD CHAIR \\ David Eisenbud \\ University of California \\ Berkeley, USA
}

\section{BOARD OF EDITORS}

Georgia Benkart

Dave Benson

Richard E. Borcherds

John H. Coates

J-L. Colliot-Thélène

Brian D. Conrad

Hélène Esnault

Hubert Flenner

Edward Frenkel

Andrew Granville

Joseph Gubeladze

Roger Heath-Brown

Ehud Hrushovski

Craig Huneke

Mikhail Kapranov

Yujiro Kawamata

János Kollár

Yuri Manin

Barry Mazur

Philippe Michel
University of Wisconsin, Madison, USA

University of Aberdeen, Scotland

University of California, Berkeley, USA

University of Cambridge, UK

CNRS, Université Paris-Sud, France

University of Michigan, USA

Freie Universität Berlin, Germany

Ruhr-Universität, Germany

University of California, Berkeley, USA

Université de Montréal, Canada

San Francisco State University, USA

Oxford University, UK

Hebrew University, Israel

University of Virginia, USA

Yale University, USA

University of Tokyo, Japan

Princeton University, USA

Northwestern University, USA

Harvard University, USA

École Polytechnique Fédérale de Lausanne
Susan Montgomery

Shigefumi Mori

Raman Parimala

Jonathan Pila

Victor Reiner

Karl Rubin

Peter Sarnak

Joseph H. Silverman

Michael Singer

Vasudevan Srinivas

J. Toby Stafford

Bernd Sturmfels

Richard Taylor

Ravi Vakil

Michel van den Bergh

Marie-France Vignéras

Kei-Ichi Watanabe

Efim Zelmanov

Shou-Wu Zhang
University of Southern California, USA

RIMS, Kyoto University, Japan

Emory University, USA

University of Oxford, UK

University of Minnesota, USA

University of California, Irvine, USA

Princeton University, USA

Brown University, USA

North Carolina State University, USA

Tata Inst. of Fund. Research, India

University of Michigan, USA

University of California, Berkeley, USA

Harvard University, USA

Stanford University, USA

Hasselt University, Belgium

Université Paris VII, France

Nihon University, Japan

University of California, San Diego, USA

Princeton University, USA

PRODUCTION

production@msp.org

Silvio Levy, Scientific Editor

See inside back cover or msp.org/ant for submission instructions.

The subscription price for 2013 is US $\$ 200 /$ year for the electronic version, and $\$ 350 /$ year $(+\$ 40$, if shipping outside the US) for print and electronic. Subscriptions, requests for back issues and changes of subscribers address should be sent to MSP.

Algebra \& Number Theory (ISSN 1944-7833 electronic, 1937-0652 printed) at Mathematical Sciences Publishers, 798 Evans Hall \#3840, c/o University of California, Berkeley, CA 94720-3840 is published continuously online. Periodical rate postage paid at Berkeley, CA 94704, and additional mailing offices.

ANT peer review and production are managed by EditFLOW ${ }^{\circledR}$ from Mathematical Sciences Publishers.

\section{PUBLISHED BY}

- mathematical sciences publishers

nonprofit scientific publishing

http://msp.org/

(C) 2013 Mathematical Sciences Publishers 


\section{Algebra \& Number Theory}

$\begin{array}{lll}\text { Volume } 7 & \text { No. } 7 \quad 2013\end{array}$

Weil representation and transfer factor

TERUJI THOMAS

Analytic families of finite-slope Selmer groups

1571

JONATHAN POTTHARST

Multiplicative excellent families of elliptic surfaces of type $E_{7}$ or $E_{8}$

ABHINAV KUMAR and TETSUJI SHIODA

Cohomological invariants of algebraic tori

SAM Blinstein and AleXANDer MerkurJeV

On abstract representations of the groups of rational points of algebraic groups and their 1685 deformations

IGOR A. RAPINCHUK

Betti diagrams from graphs

AlEXANDER ENGSTRÖM and MATTHEW T. STAMPS

Hopf monoids from class functions on unitriangular matrices

Marcelo Aguiar, NANTEl Bergeron and Nathaniel Thiem 